A N N A L E S Annales de Bretagne et des Pays de l'Ouest

\title{
Le Léon dans la Bretagne des X e -XI e siècles (Kemenet et vicomté)
}

The Léon in tenth- and eleventh-century Brittany (Kemenet and viscountcy)

Jean-Paul Soubigou

\section{(2) OpenEdition}

\section{Journals}

Édition électronique

URL : http://journals.openedition.org/abpo/2668

DOI : $10.4000 /$ abpo. 2668

ISBN : 978-2-7535-3304-2

ISSN : 2108-6443

Éditeur

Presses universitaires de Rennes

Édition imprimée

Date de publication : 30 décembre 2013

Pagination : $37-63$

ISBN : 978-2-7535-3302-8

ISSN : 0399-0826

\section{Référence électronique}

Jean-Paul Soubigou, «Le Léon dans la Bretagne des X e-XI e siècles (Kemenet et vicomté) 》, Annales de Bretagne et des Pays de l'Ouest [En ligne], 120-4 | 2013, mis en ligne le 30 décembre 2015, consulté le 01 mai 2019. URL : http://journals.openedition.org/abpo/2668 ; DOI : 10.4000/abpo.2668 


\title{
Le Léon dans la Bretagne des $\mathrm{X}^{\mathrm{e}}-\mathrm{XI}^{\mathrm{e}}$ siècles (Kemenet et vicomté)
}

\author{
Jean-Paul SouBigou \\ Chercheur indépendant ${ }^{1}$
}

\begin{abstract}
Plusieurs travaux de recherches ont été consacrés à l'histoire de la Bretagne des $\mathrm{X}^{\mathrm{e}}$ et $\mathrm{XI}^{\mathrm{e}}$ siècles ${ }^{2}$, et la présente étude a pour objet d'approfondir l'histoire du Léon au cours de la période qui va du deuxième tiers du $\mathrm{X}^{\mathrm{e}}$ siècle jusqu'à la fin du $\mathrm{XI}^{\mathrm{e}}$ siècle et qui correspond, pour les institutions civiles, à l'émergence de la société féodale, et, pour l'Église, à un début de rénovation des évêchés et monastères, désorganisés par les invasions normandes, suivie par la mise en place la Réforme grégorienne. Cette recherche va élargir le champ d'investigation à l'ensemble de la Bretagne et se baser sur l'étude d'un proto-fief spécifique à la Bretagne, appelé kemenet en breton et commendatio en latin. Le terme kemenet " désignait un fief taillé pour un vassal et confié à sa garde. La configuration du Kemenet-Ili, en Léon, aussi bien que celle du Kemenet de la Cornouaille du sud-ouest n'est pas pour le démentir ", selon B. Tanguy qui explique que leur création intervint sans-doute après le retour d'Alain Barbetorte et que l'éponyme du Kemenet-lly pourrait bien être le vicomte Hily, cité parmi les témoins d'un acte falsifié de donation au profit de l'abbaye de Redon de l'île-Grande, située au large de Perros-Guirec, par Juhel Bérenger, duc de Bretagne ${ }^{3}$. La datation de cet acte apocryphe remonte à l'époque où le donateur, Juhel Bérenger, gouvernait la Bretagne, soit le deuxième tiers du $\mathrm{x}^{\mathrm{e}}$ siècle $^{4}$. Son
\end{abstract}

1. J.-P. Soubigou est titulaire d'un DEA obtenu en 1993 à l'université de Bretagne Occidentale et consacré à l'influence des abbayes extérieures en Basse-Bretagne aux $\mathrm{XI}^{\mathrm{e}}$ et $\mathrm{XII}^{\mathrm{e}}$ siècles.

2. GuILLOTEL, Hubert, "Le premier siècle du pouvoir ducal breton (936-1040) ", Actes du 103e Congrès national des sociétés savantes, Nancy-Metz, 1978, Section de Philologie et d'Histoire, Paris, 1979, p. 63-84; Yeurc'H, Bertrand, Bourges, André-Yves, Kernevez, Patrick, "Comtes, vicomtes et lignages châtelains en Bretagne au Moyen-Age ", Bulletin de la Société archéologique du Finistère, t. cxxxvIII, 2010, p. 243-279.

3. TANGuY, Bernard, "Les pagi bretons médiévaux ", Bulletin de la Société archéologique du Finistère, t. cxxx, 2001, p. 393.

4. GUIGON, Philippe, " La donation d'Enesmur à l'abbaye de Saint-Sauveur de Redon ", dans Quaghebeur, Joëlle et Soleil, Sylvain (dir.), Le pouvoir et la foi au Moyen-Âge en 
prédécesseur, Alain Barbetorte, après s'être réfugié Outre-Manche avec d'autres compatriotes, s'était allié au roi des Francs, Louis d'Outre-Mer (avec lequel il partagea un temps l'exil), et avait mené une campagne victorieuse en Bretagne contre les Normands, vaincus en 936. Ce prince, petitfils du dernier roi de Bretagne, Alain le Grand, disparu en 908, avait reçu le soutien de l'abbé de Landévennec, Jean, et en remerciement des services rendus fit donation à l'abbaye cornouaillaise des églises de Batz-sur-Mer, et de Sainte-Croix de Nantes, entre 936 et $952^{5}$. Le duc Alain Barbetorte engagea également une rénovation des évêchés et notamment celui de SaintPol-de-Léon, en y nommant Hesdren, qui fut ensuite évêque de Nantes. On peut alors envisager qu'il ait de même entamé une réorganisation du duché, grâce au soutien du comte de Rennes, Juhel Bérenger, avec qui il avait fait alliance (Juhel est le premier témoin cité dans l'acte de donation en faveur de l'abbaye de Landévennec : "Juhel, comes ").

Nous allons examiner les kemenet recensés en Bretagne pour en tirer les premières conclusions qui seront à rapprocher des travaux récents consacrés à l'histoire de la féodalité dans l'Occident médiéval ${ }^{6}$, puis l'étude se concentrera sur le Kemenet-Ily, situé en Léon, ainsi que sur les grands lignages léonards aux $\mathrm{X}^{\mathrm{e}}$ et $\mathrm{XI}^{\mathrm{e}}$ siècles.

\section{Les kemenet : proto-fiefs bretons}

Outre le Kemenet-Ily, cinq autres fiefs ont porté le nom de kemenet en Bretagne au Moyen Âge : Kemenet d'entre Trieux et Leff, au diocèse de Tréguier; Kemenet de Porhoët en Vannes, au diocèse de Vannes; KemenetHéboé, au diocèse de Vannes; Kemenet-Guégan (scindé du précédent?); Kemenet, et Kemenet-Maen (commune de Quéménéven : détaché du précédent?), au diocèse de Cornouaille.

\section{Le Kemenet d'entre Trieux et Leff (Pommerit-le-Vicomte)}

Les premières mentions de ce fief remontent à la fin du XIII ${ }^{\mathrm{e}}$ siècle et sont contenues dans trois chartes de l'abbaye de Beauport : en 1287 (" curie domini Golovie et Kemeneti"), en 1292 (" curie domini Goelovye et Kemeneti ") et en 1296 (" cour du seigneur de Goloye et Kemeneti ") 7 . Il s'agit du seigneur de Goello et Kemenet, qui serait Henri d'Avaugour, deu-

Bretagne et dans l'Europe de l'Ouest, Mélanges en mémoire du professeur Guillotel, Rennes, PUR, 2010, p. 226-227.

5. Cartulaire de Landévennec, publié par LE MEN, René-François, et ERNAULT, Emile (éd.), Paris, 1886, rééd. Britannia Christiana-Bretagne monastique, fascicule 5/1, s.l., 1985, notice 5 , p. $562-564$.

6. DeBAX, Hélène (dir.), Vicomtes et vicomtés dans l'Occident médiéval, Toulouse, Presses Universitaires du Mirail, 2008.

7. Geslin de Bourgogne, Jean, De Barthelemy, Anatole, Anciens évêchés de Bretagne, Paris-Saint-Brieuc, 1855-1879, t. IV, p. 209 (charte de 1287) et p. 214 (charte de 1296); t. III, p. 289 (charte de 1292). 
xième du nom, descendant des comtes de Penthièvre. Une attestation plus récente de ce Kemenet est donnée dans un acte de 1598 qui cite le "seigneur de Guemenez en paroisses de Pommerit-le-Vicomte, Le Merzer, Le Faouet et autres voisines ", situées entre les rivières du Trieux, à l'ouest, et du Leff (à l'est) ${ }^{8}$. Le territoire du Kemenet d'entre Trieux et Leff recouvrait une vingtaine de paroisses appartenant à l'ancien diocèse de Tréguier et formait un triangle dont le sommet était l'embouchure commune des deux rivières (à Pontrieux et Quemper-Guézénnec) au nord et qui descendait jusqu'à Guingamp, à l'ouest, et Châtelaudren, à l'est.

Le siège primitif du Kemenet pourrait être Pommerit-le-Vicomte, qui est mentionné en 1294 dans le Livre des Ost : " visconte de Pomerit ${ }^{9}$ ". Cette paroisse était connue sous ce nom vers 1330, d'après le pouillé de l'archevêché de Tours, dans lequel elle est appelée " ecclesia de PemeritVicecomitis ${ }^{10}$ " et la toponymie semble confirmer l'implantation ancienne d'un vicomte : Kerbescond ("le village du vicomte ") et Stang-ar-Bescond ("l'étang du vicomte ") près de Pont-ar-Vonden ("le pont de la motte"). La vicomté de Pommerit pourrait ainsi être issue du démembrement du fief du Kemenet ${ }^{11}$. Dans le Livre des Ost, elle est incluse dans la baillie de Saint-Brieuc, de même que le Goello, et lorsqu'en 1212 le comte Alain réunit ses barons, afin qu'ils prêtent hommage à son jeune fils, Henri, on trouve mentionné "Guézénoc de Quemper ", qui a laissé son nom à la paroisse de Quemper-Guézénec; en raison de son rang (il est l'un des trois barons cités parmi beaucoup d'autres, selon la charte) ${ }^{12}$, on pourrait penser qu'il fut l'un des héritiers de la vicomté de Pommerit. Toutefois, le vicomte de Pommerit cité dans le Livre des Ost en 1294 a été identifié par R. Couffon comme étant Guillaume Le Chastellier ${ }^{13}$, et il est vrai que cette famille a porté le titre de vicomte de Pommerit dans des actes allant de 1329 à $1420^{14}$.

Il est intéressant de constater la coïncidence d'un kemenet avec le titre de vicomte et on peut émettre une hypothèse quant au premier titulaire en avançant le nom de "Menki, vicecomes", mentionné, comme le vicomte Hily, dans la charte de donation de l'Île-Grande à l'abbaye de Redon, dont nous avons parlé à propos du Kemenet-lly : on peut concevoir que le duc

8. Bourges, André-Yves, " Du Trieux au Leff. Toponymie et peuplement ", Bulletin et Mémoires de la Société d'émulation des Côtes-du-Nord, t. cXvIII, 1989, p. 37-40.

9. Morvan, Frédéric, La chevalerie bretonne et la formation de l'armée ducale (12601341), Rennes, PUR, 2009, p. 80.

10. Longnon, Augustin, Pouillés de la province de Tours, Paris, 1903, p. 340.

11. TANGuY, Bernard, Dictionnaire des noms de communes, trèves et paroisses des Côtesd'Armor, Douarnenez, Chasse-Marée/Ar Men, 1992, p. 240.

12. Couffon, René, "Contribution à l'étude du comté de Tréguier, Son morcellement et son remembrement partiel au Xv siècle ", Bulletin et Mémoires de la Société d'Émulation des Côtes-du-Nord, t. c, 1971, p. 40 et 41.

13. Morin, Stéphane, Trégor, Goëlo, Penthièvre. Le pouvoir des comtes de Bretagne du Xle au XII siècle, Rennes, Société d'Emulation des Côtes-d'Armor/PUR, 2010, p. 265.

14. Yeurc'H, Bertrand, Bourges, André-Yves, Kernevez, Patrick, "Comtes, vicomtes et lignages châtelains en Bretagne... ", art. cité, p. 272-273. 
Juhel Bérenger ait voulu être entouré des vicomtes en fonction dans le nord-ouest de la Bretagne lors de cet événement important ${ }^{15}$.

\section{La vicomté de Poudouvre : un ancien Kemenet?}

Il existe un lieu-dit à Sévignac (canton de Broons) portant le nom de Guéméné ${ }^{16}$. On sait que cette paroisse était traversée par une voie ancienne venant de Lamballe et appelée Sente pavée ${ }^{17}$; de même, à La ChapelleBlanche (canton de Caulnes), on relève un lieu-dit : Quemen. Le diocèse de Saint-Malo recouvrait la partie orientale du département des Côtes-d'Armor jusqu'à l'Arguenon, et plus au sud jusqu'à Sévignac, Eréac, Lanrelas, Trémorel, Merdrignac ${ }^{18}$. Qu'il y ait un lieu dit "Guéméné " en Sévignac ${ }^{19}$ est un indice intéressant, car cette paroisse appartenait au Poudouvre, pagus dont les limites étaient l'Arguenon à l'ouest et la Rance à l'est, et le Poudouvre fut un des doyennés de l'évêché de Saint-Malo (dès 1330 : Pouillé de Tours).

La dénomination de "vicomte de Poudouvre ", apparaît à la fin du $\mathrm{XII}^{\mathrm{e}}$ siècle ${ }^{20}$, mais le premier vicomte de Poudouvre pourrait être Hamon II, qui vivait vers $1081-1085^{21}$. Le nomen Hamon se retrouve à la fin du $\mathrm{X}^{\mathrm{e}}$ siècle dans le comté de Nantes. En effet, selon la Chronique de Nantes, " Haymon le vicomte ", qui aurait été tué à la bataille de Conquéreuil en 992, serait

15. Guigon, Philippe, "La donation d'Enesmur... ", art. cité, p. 226-227. Cet acte est daté de 958-960 par GulLLOTEL, Hubert, "Répertoire chronologique ", dans CHEDEVILLE, André, Guillotel, Hubert, TAnguY, Bernard, Cartulaire de l'abbaye de Saint-Sauveur de Redon, Association des amis des Archives historiques des diocèses de Rennes, Dol et Saint-Malo, Rennes, 1998-2004, t. I, p. 71-78 (p. 77).

16. Nomenclature des hameaux, écarts et lieux-dits du département des Côtes-du Nord, Liste alphabétique par communes, INSEE, Rennes, 1953, p. 137. Dans une autre commune, La Chapelle-Blanche (canton de Caulnes), on relève un lieu-dit "Quemen ".

17. TANGuY, Bernard, Dictionnaire des communes..., op. cit., p. 316.

18. Frotier de la Messeliere, Henri, "Le territoire des Côtes-du-Nord ", Bulletin et Mémoires de la Société d'émulation des Côtes-du-Nord, t. LVII, 1926, p. 25.

19. Nous avons contacté la Mairie de Sévignac et obtenu une précision de Mme Cécile Eon, adjointe au maire, dans un message du 24 février 2012, dont nous la remercions sincèrement : "le lieu Guéméné existe, mais il se prononce Quéméné ". Une visite sur les lieux a permis de constater que ce lieu-dit, "Kéméné ", est situé au bord de la Route Nationale 12, menant de Lamballe à Rennes, quelques centaines de mètres après un affluent de l'Arguenon; le secteur était anciennement bretonnisé, car en bordure de cette RN 12 se trouvent des toponymes, tels que Pengly, Pengave et la chapelle Saint-Cadroc, ou Cadoc ou Cado. La " sente pavée ", ou RN 12, traverse ensuite la paroisse de Trémeur, en direction de Rennes. Le lieu " Kéméné " pourrait marquer l'entrée dans le territoire du Kemenet, venant de Lamballe. Quant au site de "Quemen ", en La Chapelle-Blanche, il figure sur la carte de Cassini, et est également situé au bord de la voie ancienne venant de Saint-Pern et Béherel et menant vers Caulnes; c'était un endroit anciennement bretonnisé, comme l'attestent les toponymes Penhoet et Tréguéné : ce lieu Quémen pourrait remplir la même fonction que son quasi-homonyme de Sévignac.

20. Guillotel, Hubert, " Des vicomtes d'Alet aux vicomtes de Poudouvre ", Société d'histoire et d'archéologie de Saint-Malo, Annales, 1988, p. 201-215 (p. 213).

21. Geslin de Bourgogne, Jean, de Barthelemy, Anatole, Anciens évêchés, op. cit., t. 1, p. LXX-LXXII : ces auteurs voulaient reconnaître en Hamon II un vicomte de Poudouvre. 
frère de Hoel et de Guerech, par leur mère Judith, concubine d'Alain Barbetorte $^{22}$, mais Judith survécut à Alain, décédé en 952, et elle contracta, semble-t-il, un second mariage dont serait issu Haymon ${ }^{23}$. Il ne serait pas vicomte de Nantes, mais peut-être d'un des pagi du sud de la Loire; toujours est-il que ce nom appartenait au lignage des vicomtes d'Alet ${ }^{24}$.

Du Paz, auteur du début du XVII ${ }^{\mathrm{e}}$ siècle, désigne ce lignage comme étant celui des vicomtes de Dinan, dont le premier représentant serait Ammon, qui vivait du temps du duc Geoffroy (décédé en 1008) et au début du règne de son fils Alain III, et qui eut plusieurs fils, dont l'aîné porta le nom de Hamon ${ }^{25}$. Cette généalogie est confirmée par les historiens modernes, qui l'appellent toutefois du nom de vicomtes d'Alet. Ainsi, le lignage des vicomtes de Poudouvre a été étudié récemment par C. Amiot, qui indique que la branche aînée des vicomtes d'Alet, devenus vicomtes de Poudouvre au $\mathrm{XII}^{\mathrm{e}}$ siècle, contrôlait le territoire entre l'Arguenon et la Rance et qu'elle possédait plusieurs lieux fortifiés : le château du Plessis-Balisson (près de Ploubalay) fut dès le XII ${ }^{\mathrm{e}}$ siècle une place forte des vicomtes de Poudouvre et celui de Montafilan, à Corseul, peut correspondre à un bien patrimonial de ce lignage vicomtal, avant de devenir celui d'une des branches de la maison de Dinan.

Il est intéressant de noter les liens entre ce puissant et ancien lignage et l'abbaye de Saint-Jacut-de-la-Mer, située en Poudouvre, qui possédait une dîme à Corseul, et qui avait bénéficié de plusieurs autres donations, notamment à Dinan ${ }^{26}$. Elle relevait de l'évêché de Dol-de-Bretagne, dont elle constituait une enclave dans celui d'Alet (Saint-Malo), et était déjà influente au début du $\mathrm{XI}^{\mathrm{e}}$ siècle, car son abbé, Hynguethen, se vit confier par le duc de Bretagne la mission de restaurer le monastère de Saint-Méen-de-Gaël, entre 1024 et $1034^{27}$. À Dinard existait une motte sur la pointe qui a gardé le nom de la Vicomté, dominant l'embouchure de la Rance ${ }^{28}$, et la paroisse de La Vicomté-sur-Rance rappelle peut-être l'existence d'un fief plus ancien et " semble garder dans sa micro-toponymie la mémoire d'une ancienne résidence des vicomtes d'Alet ${ }^{29} »$. Le titre vicomtal paraît avoir été relevé plus

22. La chronique de Nantes, Merlet, René, (éd.) Paris, 1896, p. 128 et 132 : ce texte précise : " non pas d'un même père mais de leur mère Judith ".

23. QuAGHEBEuR, Joëlle, "Alain Barbe-Torte ou le retour improbable d'un prince en sa terre ", Bulletin de l'Association bretonne, t. cXII, 2003, p. 143-168 (p. 160).

24. CHÉDEVILLE, André, "Un millénaire : la bataille de Conquereuil (992) et sa place dans l'histoire politique et militaire de la Bretagne ", Mémoires de la Société d'histoire et d'archéologie de Bretagne, t. Lxx, 1993, p. 365-384 (p. 371).

25. Du PAZ, Augustin, Histoire généalogique de plusieurs maisons illustres de Bretagne, Paris, 1619, I-15.

26. Gullotel, Hubert, "Des vicomtes d'Alet aux vicomtes de Poudouvre », art. cit., p. 209.

27. DE LA BORDERIE, Arthur, Recueil des actes inédits des ducs et princes de Bretagne (XI XII et XIII siècles), Rennes, 1888, p. 4.

28. Baneat, Paul, Le département d'Ille-et-Vilaine Histoire-Archéologie-Monuments, Mayenne, Éditions Régionales de l'Ouest, (rééd.), 1994, t. I, p. 472.

29. Morin, Stéphane, Trégor, Goëlo, Penthièvre Le pouvoir des comtes de Bretagne du XI au XIII siècle, op. cit., p. 110, 111, et note 325 : " des structures gallo-romaines et le lieu- 
tard par la famille de La Bellière, nom du château implanté dans la paroisse de la Vicomté-sur-Rance, famille qui à la fin du XIII ${ }^{\mathrm{e}}$ siècle s'intitulait vicomte de Dinan, puis vicomte de La Bellière ${ }^{30}$.

Ne peut-on envisager que le Poudouvre ait été confié à un vicomte dans la seconde moitié du X ${ }^{\mathrm{e}}$ siècle, comme le Quemenet Ily, que le fief d'origine soit resté à l'une des branches du lignage vicomtal (l'aînée?), et que les autres branches aient installé leur pouvoir à Dinan, Dol et Combourg au cours du $\mathrm{XI}^{\mathrm{e}}$ siècle? Le lignage des vicomtes de Poudouvre (ou de Dinan ou d'Alet) pourraient descendre d'un vicomte Hamon, vivant dans la deuxième moitié du $\mathrm{X}^{\mathrm{e}}$ siècle, qui aurait été installé par Alain Barbetorte ou par le seigneur qui a dominé le nord-est du duché de Bretagne après la disparition de ce dernier, en 952, à savoir Wicohen, qui cumulait en outre le titre d'archevêque de Dolde-Bretagne. Wicohen/luthoen, qui fut contemporain d'Alain Barbetorte (il souscrit à la charte de donation de Batz-sur-Mer à l'abbaye de Landévennec) a dominé la scène bretonne après le décès de ce dernier en 952, exerçant un contre-pouvoir face au nouveau duc, Juhel Bérenger ${ }^{31}$. Il s'était allié au comte d'Anjou, Foulques Le Bon, tout comme le vicomte Hamon qui fut tué à la bataille de Conquereuil en 992, opposant le comte d'Anjou au duc de Bretagne, Conan ${ }^{32}$. La constitution d'un proto-fief dans la partie occidentale de l'évêché d'Alet, confié à un vicomte, pouvait conforter la mainmise d'un fidèle d'Alain Barbetorte sur tout le nord-est de la péninsule : ce lignage a d'ailleurs été appelé vicomte de Dol, comme l'atteste la désignation de vicomtesse de Dol, donnée à l'épouse de Hamon I au début du XI' siècle $^{33}$.

\section{Le fief de Kemenet ou Porhoët en Vannes (jouxtant Josselin)}

C'est la désignation donnée par M. Rozensweig : "Au XV siècle, le comté de Porhoët se divisait en deux châtellenies principales, celle de Josselin et celle de La Chèze. Après 1603, ce comté, réduit à la châtellenie de Josselin, renfermait une vingtaine de paroisses (correspondant aux cantons de Josselin et de Saint-Jean-Brévelay). Celles de ces paroisses qui étaient situées sur la rive droite de l'Oust, dans le diocèse de Vannes, formaient une subdivision particulière, désignée sous le nom de baillage d'Outre l'Eau, fief de Kemenet, ou Porhoët-en-Vannes ${ }^{34}$."

dit La vieille-Vicomté pourraient indiquer un ancien site de peuplement contrôlé vers le $\mathrm{XI}^{\mathrm{e}}$ siècle par les vicomtes d'Alet ".

30. Guillotel, Hubert, "Des vicomtes d'Alet aux vicomtes de Poudouvre ", art. cit., p. 213-214.

31. GuILLOTEL, Hubert, " Le premier siècle du pouvoir breton ", art. cit., p. 77.

32. Chedeville, André, et Tonnerre, Noël-Yves, La Bretagne féodale (XI ${ }^{e}$-XII ${ }^{e}$ siècles), Rennes, Éditions Ouest-France Université, 1987, p. 30 à 35.

33. SOUBEn, Patrick, et BRAND'HONNEur, Michel, «Les enjeux de pouvoir autour de l'ancien domaine monastique carolingien de Saint-Bern ", dans QUAGHEBEUR, Joëlle, et SoLEIL, Sylvain (dir.), Le pouvoir et la foi au Moyen-Age en Bretagne et dans l'Europe de l'Ouest, Mélanges en l'honneur du professeur Hubert Guillotel, Rennes, PUR, 2010, p. 486, 487.

34. RoSENZWEIG, Louis, Dictionnaire topographique du département du Morbihan, comprenant les noms de lieux anciens et modernes, Paris, Sociétété polymatique du Morbihan, 1890, p. XXI. 
Le fief de Kemenet existait dans la première moitié du $\mathrm{XI}^{\mathrm{e}}$ siècle, selon une charte de l'abbaye de Redon, datable des années 1041-1062, dans laquelle le vicomte Josselin, après la mort de son père, le vicomte Guéthenoc, donna à l'abbé Pérennes " le couvent de Sainte-Croix avec le vieux faubourg, jusqu'au milieu de la rivière Oust [...]. Il donna dans son fief les villes de Crannam et de Tinsedio [...], Plucgaduc en Keminet ". Il s'agit de la paroisse de Pleucadeuc, située rive droite de l'Oust au sud-est de Josselin. Dans un autre acte du cartulaire, un chevalier de la paroisse de Sérent fait don à l'abbaye de Redon de diverses dîmes, dont celles de Kemenet (charte de 1120 environ) ${ }^{35}$. La toponymie n'a conservé qu'un Quémené, à Guéhenno, lieu-dit où existait un moulin à eau ${ }^{36}$. Ce Kemenet comprenait une vingtaine de paroisses, ce qui lui donnait les dimensions d'un fief comme le Kemenet Guéguan, selon G. Bernier ${ }^{37}$. Il était entièrement situé dans le diocèse de Vannes, et délimité au sud par la Claie, affluent de l'Oust, qui rejoignait cette dernière en aval de Malestroit.

L'origine des vicomtes dits de Porhoët est double : Guethenoc, par son nom et les biens qu'il possède, se rattache au bassin de l'Oust, et ce lignage pouvait être anciennement implanté autour de Josselin, car un riche alleutier du nom de Guethenoc est cité à plusieurs reprises dans le cartulaire de Redon au $\mathrm{IX}^{\mathrm{e}}$ siècle, comme en 857 à Caro. Mais, en tant que vicomte de Rennes, Guethenoc serait lié à l'aristocratie de la Loire moyenne, comme semblent l'attester les noms donnés à ses descendants, Josselin et Eudes ${ }^{38}$.

La famille de Guethenoc est d'origine vannetaise et peut-être machtiernale ${ }^{39}$. Vers 952-960, dans une charte-notice établie par Juhel Bérenger, duc de Bretagne, en faveur de Landévennec, confirmant la donation d'un bien situé dans la vicaria de Carentoir, "in pago Brouuerec ", figure parmi les témoins "Uuethenoc ", sans titre ${ }^{40}$. Récemment, la thèse qui fait de Guéthénoc un vicomte de Rennes a été remise en cause, car ce titre n'aurait été acquis que par son fils, Josselin, dans le deuxième quart du XI ${ }^{\mathrm{e}}$ siècle, période pendant laquelle ce dernier est mentionné parmi les premiers témoins dans quatre

35. Cartulaire de l'abbaye de Saint-Sauveur de Redon, Chedeville, André, Guillotel, Hubert, TANGUY, Bernard, Association des amis des Archives historiques des diocèses de Rennes, Dol et Saint-Malo, op. cit., t. I, $\mathrm{f}^{\circ} 141 \mathrm{r}^{\circ}$, et f ${ }^{\circ} 183 \mathrm{v}^{\circ}$, et p. 71 à 78 : datation par GUILLOTEL, Hubert.

36. RosenZWEIG, Louis, Dictionnaire topographique du Département du Morbihan..., op. cit., p. 226.

37. BERNIER, Gildas, Les chrétientés bretonnes continentales de l'origine à 845, Thèse dactylographiée présentée en vue du doctorat d'Université devant l'Université de HauteBretagne, Rennes, 1980, p. 148, et carte p. 147.

38. Tonnerre, Noël-Yves, Naissance de la Bretagne, Géographie historique et structures sociales de la Bretagne méridionale (Nantais et Vannetais) de la fin du VIII à la fin du XII siècle, Angers, Presses Universitaires d'Angers, 1994, p. 356.

39. BouRRIQUEN, Arnaud, Les vicomtes de Rennes et leurs descendants, les Porhoët/Rohan, face au pouvoir (fin $X^{e}$-fin $X I^{e}$ siècle), mémoire de master 2 d'histoire, dactyl., Université de Rennes 2, 2008, p. 6-18.

40. Chedeville, André, " La bataille de Conquereuil... ", art. cité, p. 373. 
chartes établies par la famille comtale de Rennes ${ }^{41}$ : il est vrai que Guéthénoc, père de Josselin, n'est pas désigné formellement comme vicomte dans deux actes de donation en faveur de l'abbaye du Mont-Saint-Michel, datables de $990 / 992^{42}$. Toutefois, dans la charte de Saint-Sauveur de Redon relatant la fondation du château de Josselin, qui précise la fonction vicomtale de Guéthénoc, comme dans d'autres chartes réécrites de cette abbaye, on peut considérer que les moines reprenaient correctement les noms et qualités des témoins (" vicomte Ily " et " vicomte Menki ", dans la charte de donation de l'île d'Enesmur, supra); de même, dans la charte cCCLVI, qui mentionne les vicomtes Guéthénoc et Guiomarch, aux côtés du duc Alain III et du comte de Cornouaille Alain Canhiart. Enfin, on pourrait penser que, s'agissant d'une abbaye (celle du Mont-Saint-Michel) qui s'émancipait de la tutelle bretonne, la mention du titre vicomtal avait moins de poids que pour Saint-Sauveur de Redon. Quoi qu'il en soit, le lignage des seigneurs dits de Porhoet apparaît comme étant lié à la famille des comtes de Rennes, depuis Juhel Béranger jusqu'au duc Conan II, durant près d'un siècle.

Le lignage de Guéthénoc peut-il être associé à la création du Kemenet? On peut penser en tout cas qu'il était possessionné depuis le début du $\mathrm{XI}^{\mathrm{e}}$ siècle au moins sur la rive droite de l'Oust, car dans la charte datable de 1041-1062 en faveur de l'abbaye de Redon, Josselin fait don du « couvent de Sainte-Croix et des saints martyrs Corneille et Cyprien, avec un vieux faubourg ", situés rive droite de la cité de Josselin (sur le territoire de la paroisse de Guégon), mais aussi Pleucadeuc en Kemenet. Le château de Josselin se trouvait au croisement des voies Rennes (Condate)/Castel-Noë et Vannes/Merdrignac (et Corseul). La fondation de Josselin a été comparée par H. Guillotel à celle de Châteaubriant, près de laquelle se trouvaient les restes d'un ancien monastère remontant à la période mérovingienne, et où fut élevé, au milieu du $\mathrm{XI}^{\mathrm{e}}$ siècle, un château auquel fut donné le nom de son bâtisseur, Brien ${ }^{43}$. Il est vraisemblable que le " vieux bourg " de SainteCroix existait au $\mathrm{x}^{\mathrm{e}}$ siècle, voire antérieurement en raison de la dédicace du sanctuaire à des saints martyrs, et que cette agglomération située au croisement de voies antiques ait pu constituer le siège du Kemenet. À partir du XII ${ }^{\mathrm{e}}$ siècle, c'est la branche cadette, celle des vicomtes de Rohan, qui a porté le titre de vicomte, après que la branche aînée se soit fondue dans la maison de Fougères, au XIII ${ }^{\mathrm{e}}$ siècle, en conservant sous son fief une douzaine de paroisses situées sur la rive sud ${ }^{44}$ correspondant au Kemenet, et

41. BourRIQUEN, Arnaud, "Les origines de Pontivy et les vicomtes de Rohan aux XII et $\mathrm{XIII}^{\mathrm{e}}$ siècles ", Actes du Congrès de Pontivy, Mémoires de la Société d'histoire et d'archéologie de Bretagne, t. LXXXVIII, 2010, p. 31.

42. Boute, Pierre, Desbordes, Olivier (éd.), Chroniques latines du Mont-Saint-Michel (IX ${ }^{\text {- }}$ XII siècles) Les manuscrits. Textes fondateurs, Caen, Presses universitaires de Caen, 2009, p. 383-384.

43. Guillotel, Hubert, "De la vicomté de Rennes à la vicomté de Porhoet (fin $\mathrm{X}^{\mathrm{e}}$-milieu XII ${ }^{\mathrm{e}}$ siècle) ", Mémoires de la Société d'histoire et d'archéologie de Bretagne, t. LXXII, 1995, p. 18-19.

44. Chedeville, André, Tonnerre, Noël-Yves, La Bretagne féodale..., op. cit., p. 158. 
qui formaient aux XVII ${ }^{\mathrm{e}}$ et XVIII ${ }^{\mathrm{e}}$ siècles le " baillage d'Outre-Eau, ou fief de Kemenet ou Porhoët-en-Vannes ».

\section{Kemenet-Heboë et Kemenet-Guégan}

Ces deux fiefs étaient compris entre le Blavet et l'Ellé et correspondaient à la partie occidentale du diocèse de Vannes : le Kemenet-Héboé était délimité au sud par la mer (et incluait l'lle de Groix) et au nord était limitrophe du Kemenet-Guégan, mais la frontière entre les deux n'était pas naturelle ${ }^{45}$. Le Kemenet-Héboé apparaît dans les sources documentaires vers le milieu du XI ${ }^{\mathrm{e}}$ siècle dans un acte du cartulaire de Quimperlé où figurent des témoins de haut rang, les uns cornouaillais, les autres du KemenetHéboé, dont Huelin, fils de Bérenger ${ }^{46}$. Ce dernier patronyme pourrait suggérer une alliance avec la famille comtale de Rennes, dans laquelle il fut porté par Juhel Bérenger, comte de Rennes, puis duc de Bretagne à la suite d'Alain Barbetorte, et qui vivait vers le milieu du $\mathrm{X}^{\mathrm{e}}$ siècle. Mais, certains auteurs préfèrent reconnaître dans ce lignage la maison vicomtale de Vannes, qui aurait installé au début du XI ${ }^{\mathrm{e}}$ siècle le siège de son fief au Vieil-Hennebont ${ }^{47}$. Quant au Kemenet Guégan, dont le nom se retrouve dans la cité de Guéméné-sur-Scorff, il pourrait être un démembrement d'un Kemenet original : il porte le nom de son premier titulaire, qui rappelle celui du fils de Huelin, Guégon. Le titre vicomtal n'était pas porté par ce lignage, mais a pu échoir à la fin du XII ${ }^{\mathrm{e}}$ siècle à une branche cadette, celle des vicomtes dits de "Poher ", qui se serait installée dans cette dernière région à la faveur d'une alliance matrimoniale ${ }^{48}$. Ainsi, le titre vicomtal appartenant à l'origine aux seigneurs du Kemenet Héboé, eux-mêmes issus d'un vicomte de Vannes, se serait transmis à un rameau connu comme étant vicomtes de Poher.

Une autre approche des origines de ce proto-fief pourrait être proposée. En effet, le Kemenet du vannetais occidental fut confié à un personnage qui serait nommé Héboé, mais que nous sommes tenté de le rapprocher de Nuuenoé, et Kemenet Heboé serait une forme corrompue de KemenetNuuenoé (Keme/Névénoé, par contraction, et le " $\mathrm{t}$ " n'étant pas prononcé, comme dans Guéméné). Le nomen de Nuuenoé était celui d'un personnage appartenant à l'entourage d'Alain Barbetorte, car cité dans la charte de donation de l'église de Batz-sur-Mer à l'abbaye de Landévennec, vers 940952 : il figure parmi les témoins entre les deux fils du duc et deux vicomtes, Jestin et Dilés. Ce dignitaire est mentionné, sous la forme Numinoe, dans

45. Tonnerre, Noël-Yves, Naissance de la Bretagne..., op. cit., p. 359-361.

46. MAîTRE, Léon, De Berthou, Paul, Cartulaire de l'abbaye de Sainte-Croix de Quimperlé, Paris/Rennes, s.d., p. 112.

47. Quaghebeur, Joëlle, La Cornouaille du IX e au XII siècle Mémoire, pouvoirs, noblesse, Quimper, Société archéologique du Finistère, 2001, p. 126, note 96; GuILLOTEL, Hubert, "Administration et finances ducales sous le règne de Conan III ", Mémoires de la Société d'histoire et d'archéologie de Bretagne, t. LXVIII, 1991, p. 32.

48. QuagheBeur, Joëlle, op. cit., p. 381-382. 
la charte 40 du même cartulaire comme l'un des signataires de la donation effectuée par Juhel Bérenger, quelques années plus tard, d'un bien de la paroisse de Carentoir, située en Brouuerec, charte dans laquelle il porte le titre de comte ${ }^{49}$. Si cette supposition était avérée, elle expliquerait que le lignage n'ait pas porté le titre de vicomte, car étant issu d'une maison comtale.

Si cette hypothèse ne pouvait être retenue ${ }^{50}$, on peut conclure que :

- le premier titulaire authentifié s'appelait Bérenger, qui vivait vers 1029-1037, ce qui conforte l'hypothèse d'une proximité avec le duc Juhel Bérenger;

- Huelin (décédé entre 1037 et 1066), épousa Avan, sœur du comte de Cornouaille, Alain Canhiart : cette alliance semble confirmer le rang des seigneurs du Kemenet au sein de l'aristocratie bretonne au XI ${ }^{\mathrm{e}}$ siècle;

- son fils Guégon (décédé vers 1069), et son petit-fils, Tanki (décédé entre 1096 et 1114) sont ses descendants connus.

$\mathrm{Au} \mathrm{XIII}$ e siècle, cette seigneurie fut divisée en trois fiefs et le nom disparut de ce fait ${ }^{51}$.

\section{Kemenet de Cornouaille et Kemenet-Maen}

Il pourrait s'agir, comme les précédents, d'un seul fief, à l'origine, comme le suggère la géographie. Les seigneurs de Léon, et avant les vicomtes de Léon, possédaient, outre le Kemenet, Coet-Squirriou, motte féodale située sur le territoire de la paroisse de Quemeneven, limitrophe de Locronan, au nord-est. En effet, à la fin de l'Ancien Régime, le Kemenet s'étendait sur onze paroisses situées entre la montagne de Locronan, la rive droite de l'Odet, la rivière d'Audierne et la mer. Ainsi, Quéménéven (Kemenet-Maen, dans le compte de 1368 du pouillé de Tours, pour le diocèse de Cornouaille ${ }^{52}$ ) se trouvait séparée du Kemenet par le territoire de Locronan, prieuré de l'abbaye de Sainte-Croix de Quimperlé. Le siège du Kemenet se trouvait à Penhars (jouxtant Quimper) d'après une déclaration au roi datée de $1681^{53}$.

49. Cartulaire de Landévennec, publié par LE MENN, René-François, et ERNAULT, Emile, rééd. Britannia Christiana-Bretagne monastique, op. cit., charte 25, p. 564; charte 40, p. 569.

50. Nous avons sollicité DesHAYEs, Albert, qui par courrier du 20 janvier 2012, nous a aimablement donné l'étymologie de Héboé : "Ce nom comprend un élément -boe au sens de "coup" et par extension "frappeur" avec le préfixe he- évolué du vieux breton ho-, préfixe superlatif. Cet élément boe-se retrouve aussi dans le nom du premier roi de Bretagne Erispoé, mais sous une forme renforcée. "

51. Chedeville André, et TonNerre, Noël-Yves, La Bretagne féodale..., op. cit., p. 170-172.

52. Longnon, Augustin, Pouillés de la province de Tours, op. cit., p. 306.

53. De la BorderIE, Arthur, Histoire de la Bretagne, t.III, Rennes/Paris, 1899, p. 77, note 1 : "Herveus de Leonia, filius Margiliae, dat R. filio cari senescalliam hereditaram [...] i $n$ quinque plebibus an Kemenet, in Plebe Cuvan (Pluguffan), in Plebe Merin (Plomelin), in Plebe Castelli (Plogastel), in Plebe Nidi (Plonéis), in Capite Arth (Penhars), et in Nemus Schyrriou (Coet-Squirriou). Anno MCCVIII ": Extrait de la Généalogie des vicomtes de Léon, par Dom LOBINEAU, à la suite du Traité historique des Barons de Bretagne, Bibl. mun. Rennes, ms. 498, p. 324. Et p. 77, note 2. 
D’après G. Bernier, c'est Kaer-Huel en Plomelin, cité dans la charte de Hoël (Kaer Uhel in Kemmenet) et qui serait Ker-Huel-Bodivit, dans la seigneurie de Bodivit ${ }^{54}$.

Selon H. Bourde de la Rogerie, la plupart des seigneuries de la Cornouaille occidentale paraissent avoir été d'anciennes dépendances du Kemenet, mais des cessions nombreuses l'avaient modifié avant le $\mathrm{XVII}^{\mathrm{e}}$ siècle $^{55}$. À la suite de nombreux démembrements, le Kemenet n'aurait conservé au XIII ${ }^{\mathrm{e}}$ siècle qu'une douzaine de paroisses.

Le vicomte Diles était possessionné dans le Cap-Caval, au sud du Kemenet, d'après une charte de Landévennec, dans laquelle il fait plusieurs donations de biens venant de l'héritage de ses parents, notamment dans les paroisses de Plonéour-Lanvern, Beuzec, Pouldreuzic et Peumeurit. Ce vicomte fut témoin de la donation de l'église de Batz-surMer à Landévennec par le duc Alain Barbetorte, relatée par la charte 25 du cartulaire de l'abbaye (supra) vers 940-950, et il est appelé Diles Heirguor Chebre (Cambrie, désignant le Pays de Galles) dans la généalogie des comtes de Cornouaille incluse dans le même cartulaire (notice 54), ce qui laisse entendre qu'il avait accompagné le duc dans son exil en Angleterre, après les invasions normandes du deuxième quart du $\mathrm{x}^{\mathrm{e}}$ siècle. Il précède dans cette liste Budic, premier comte de Cornouaille historiquement authentifié, qui donna également des villae situées à PlonéourLanvern et à Plozévet (notice 45). On peut envisager que le vicomte Diles se vit confier par Alain Barbetorte un pouvoir de commandement dans cette partie de la Cornouaille, après que les envahisseurs normands en aient été chassés ${ }^{56}$. Enfin, une alliance entre Dilès et le lignage de Budic Castellin serait à l'origine de la maison des comtes de Cornouaille, à la fin du $\mathrm{X}^{\mathrm{e}}$ siècle $^{57}$.

\section{Premières conclusions sur les kemenet}

La géographie historique des kemenet n'est connue que par des documents tardifs, à savoir principalement le Pouillé de l'archevêché de Tours, établi vers 1330 pour l'ensemble des diocèses qui en relevaient, dont ceux du duché de Bretagne. En effet, l'archidiaconé de Kemenet-Ily et les doyennés de Poudouvre, de Kemenet-Héboé et de Kemenet-Guégan ont existé : ainsi, l'énumération des paroisses appartenant à ces subdivisions religieuses a permis d'en établir les contours, qui correspondent à des territoires délimités par des cours d'eau; d'ailleurs, la signification de Poudouvre est, en langue bretonne : "pays d'entre deux eaux ", " paou

54. BERNIER, Gildas, Les chrétientés bretonnes continentales..., op. cit., p. 150.

55. Bourde DE LA RogerIE, Henri, "Liste des juridictions du présidial de Quimper ", Bulletin de la Société archéologique du Finistère, t. LVII, 1930, p. 107-110.

56. Soubigou, Jean-Paul, " Recherches sur les origines du Kemenet de Cornouaille (IX ${ }^{\mathrm{e}}$ $\mathrm{XI}^{\mathrm{e}}$ siècles) ", Annales de Bretagne et des Pays de l'Ouest, t.115, mars 2008, n 1, p. 104-105.

57. QuagheBeur, Joëlle, La Cornouaille du IX $X^{e}$ au XII siècle, op. cit, p. 111-112. 
daou dour ${ }^{58}$ ". Ils étaient donc délimités par des rivières, qui constituent des limites naturelles propices à la détermination de conscriptions militaires ou religieuses ${ }^{59}$. En outre, il y avait coïncidence entre les limites des Kemenet et celles de certains anciens diocèses : ainsi le Leff séparait les diocèses de Tréguier et de Saint-Brieuc, l'Arguenon ceux de Saint-Brieuc et d'Alet, l'Oust était la limite entre les évêchés d'Alet et de Vannes, et enfin l'Ellée constituait la frontière entre les diocèses de Vannes et de Cornouaille.

En Bretagne, l'érection du siège de Dol en archevêché avait été suivie avant la fin du $\mathrm{x}^{\mathrm{e}}$ siècle par la création de deux nouveaux diocèses, portant leur nombre à neuf. Il est vraisemblable que Wicohen/Iuthoen, qui, à partir de 944, porte le titre d'archevêque dans les actes dont il est témoin et d'évêque des Bretons dans ceux qu'il souscrit, prit une part active à cette réorganisation. En 970, le pape Jean XIII informe les comtes bretons de la plainte que lui a adressée l'archevêque de Tours au sujet de l'usurpation des droits de son archevêché par leur archevêque (i.e. Wicohen), usurpation qui pourrait être interprétée comme étant la fondation de nouveaux évêchés ne dépendant pas de Tours ${ }^{60}$; en 990 , dans la charte du duc Conan en faveur de l'abbaye du Mont-Saint-Michel, les neuf prélats bretons sont témoins, dont Main successeur de Wicohen sur le siège de Dol.

La fixation définitive des limites des évêchés ne s'est réalisée, en Bretagne, comme dans les autres régions de l'Occident, qu'au XI ${ }^{\mathrm{e}}$ au plus tôt ${ }^{61}$, notamment pour celui de Tréguier, constitué aux dépens du diocèse de Léon, et elle a donné lieu à des conflits qui transparaissent dans certaines sources hagiographiques, comme dans les trois Vitae de saint Tugdual, rédigées à Tréguier durant le $\mathrm{xI}^{\mathrm{e}}$ siècle $^{62}$. Ainsi, il est possible d'en déduire que des frontières préexistantes ont été utilisées pour la délimita-

58. Lunven, Anne, Approches historique et archéologique de la fondation des territoires ecclésiastiques (diocèse, paroisse et cadres intermédiaires) entre le ve et le XIII siècle, thèse de doctorat d'histoire dactylographiée, sous la direction de PIсHOT, Daniel, Université de Rennes 2, 2012, p. 374 : " malgré les variations tardives enregistrées dans la géographie des doyennés, les comptes de 1330 ont été utilisés pour reconstituer l'organisation archidiaconale et décanale "

59. MAZEL, Florian, "L'espace du diocèse dans les cartulaires cathédraux ", dans MAZEL, Florian (dir.), L'espace du diocèse Genèse d'un territoire dans l'Occident médiéval ( $V^{e}$-XIII ${ }^{e}$ siècle), Rennes, PUR, 2008, p. 375 : "L'utilisation du réseau hydrographique semble à la fois la plus évidente et la plus étonnante. La plus évidente car fleuves et rivières, comme sommets et lignes de crêtes, servent fréquemment de repères, voire de frontières, dans les processus de délimitation domaniaux ou politiques. "

60. GUILLOTEL, Hubert, "Le premier siècle du pouvoir ducal breton ", art. cit., p. 77.

61. MAZEL, Florian, " Pouvoirs seigneuriaux et territoires diocésains ", dans DEBAX, Hélène (dir.), Vicomtes et vicomtés dans l'Occident médiéval, Toulouse, Presses universitaires du Mirail, 2008, p. 225 : «La projection spatiale du pouvoir épiscopal s'appuie sur des cadres laïques, c'est-à-dire les pouvoirs vicomtaux, qui eux-mêmes s'appuient sur la topographie des vallées : ce sont les seuls cadres territoriaux effectifs au milieu du $\mathrm{XI}^{\mathrm{e}}$ siècle. D'ailleurs, comme en Provence, il faut attendre, la fin du XII ${ }^{\mathrm{e}}$ siècle ou le XIII ${ }^{\mathrm{e}}$ siècle pour que les limites entre diocèses soient définitivement établies. "

62. Merdrignac, Bernard, Plouchart, Louisa, "La fondation des évêchés bretons. Questions de l'histoire religieuse à la géographie sociale ", dans MAZEL, Florian (dir.), 
tion des territoires diocésains, dont celles des kemenet, entités qui sont attestées dès la seconde moitié du XI ${ }^{\mathrm{e}}$ siècle. Mais, aucun siège épiscopal ne se trouvait sur le territoire des kemenet, et on peut penser que les évêques avaient organisé leur défense en complément des pouvoirs laïcs.

En conclusion, l'étymologie de kemenet tend à suggérer une acception plus territoriale que fonctionnelle : le terme proviendrait du verbe " comyna " (découper, détacher, en vieux breton) et désignerait un territoire " découpé " et placé sous le commandement d'un seigneur, la traduction latine étant " commendatio ${ }^{63}$ ". L'utilisation d'un mot breton jusqu'aux confins de l'évêché de Vannes et donc de l'aire bretonnisée témoigne enfin de l'ancienneté de ce proto-fief. La répartition géographique des sept Kemenet recensés en Bretagne ${ }^{64}$ tend à suggérer qu'ils ont été constitués pour organiser et quadriller militairement la principauté bretonne; elle devait permettre également aux ducs (Alain Barbetorte, puis Juhel Bérenger, son successeur) de se rendre par étapes dans toutes les parties de la péninsule, à partir de Nantes ou de Rennes. On a pu constater qu'ils avaient, sauf celui de Porhoët-en-Vannes, une frontière maritime dans le but vraisemblable d'assurer la défense des côtes du duché. Ils avaient en outre pour mission de contrôler certains cours d'eau qu'avaient utilisés les Vikings pour pénétrer dans les terres.

Il semble que certains kemenet aient été confiés à des personnages qui ont porté le titre de vicomte : ce fut le cas du vicomte Ily, en Léon, et vraisemblablement de Dilès en Cornouaille, témoin de la charte de donation faite par le duc Alain Barbetorte en faveur de l'abbaye de Landévennec aux côtés d'un autre vicomte, Jestin, et peut-être du vicomte Mainki en TrégorGoëllo, qui est mentionné dans l'acte de donation par Juhel Bérenger de l'Île-Grande en faveur de l'abbaye de Redon (on peut faire un parallèle entre la charte de Landévennec et celle, postérieure, de Redon, car les ducs donateurs y sont entourés de deux vicomtes). Il n'est pas attesté que les seigneurs de Porhoët aient porté le titre vicomtal dès fin du $\mathrm{X}^{\mathrm{e}}$ siècle, avant de devenir les vicomtes attitrés des comtes de Rennes au XI ${ }^{\mathrm{e}}$ siècle. L'équivalence entre kemenet et vicomté se retrouverait dans le fief des vicomtes de Poudouvre (ou de Dinan ou d'Alet). En tous cas, on recense, entre 940 et 970 environ, quatre vicomtes dans l'entourage des deux ducs

L'espace du diocèse. Genèse d'un territoire dans l'Occident médiéval ( $V^{e}$-XIII siècle), Rennes, PUR, 2008, p. 160-161.

63. Yeurc'H, Bertrand, Bourges, André-Yves, Kernevez, Patrick, "Comtes, vicomtes et lignages châtelains en Bretagne au Moyen-Âge ", art. cit, p. 265.

64. Ce nombre s'obtient en comptant deux Kemenet vannetais : Kemenet-Héboé et Kemenet-Guégan, mais il ne serait que de six en optant pour un seul Kemenet vannetais, à l'origine; il faudrait donc, pour avoir le chiffre symbolique de sept, rechercher un autre Kemenet, qui pourrait se trouver dans le diocèse de Nantes, et on pense à Guéméné-Penfao; mais, l'étymologie de ce Guéméné ne serait pas kemenet ; toutefois, le territoire compris entre les estuaires de la Vilaine et de la Loire aurait pu constituer une circonscription de défense, comparable aux kemenet, d'autant qu'un lignage vicomtal y est attesté, à Donges, depuis le milieu du $\mathrm{XI}^{\mathrm{e}}$ siècle, lignage qui a porté le titre de vicomte de Nantes : TonNERRE, Noël-Yves, op. cit., p. 335-337. 
qui se sont succédés pendant cette période, ce qui pose la question de leur éventuelle implantation territoriale.

Le vicomte était l'unique " lieutenant " du comte dans l'organisation carolingienne, et il avait pour mission de suppléer le comte dans toutes ses fonctions. Dans la seconde moitié du $\mathrm{x}^{\mathrm{e}}$ siècle, les mentions de vicomtes dans les textes semblent se multiplier, et leur localisation se faire plus précise. Dans le dernier quart de ce siècle, les informations deviennent suffisamment fournies pour qu'on puisse suivre de véritables lignées ${ }^{65}$. La situation du duché de Bretagne dans la période qui nous occupe peut être comparée à celle du comté du Poitou quelques décennies plus tôt. En effet, à partir de 902, le comte Elbe Manzer engage une profonde réorganisation de l'administration comtale, et de 902 à 935, cinq vicomtes différents sont cités dans les textes, et on assiste à un début de processus de patrimonialisation et de territorialisation, avec l'apparition du terme " in suo vicecomitatu "; après cette phase très active de création vicomtale, seuls subsistent au milieu du $\mathrm{x}^{\mathrm{e}}$ siècle les vicomtes de Thouars, de Chatellerault et d'Aulnay, qui sont détenteurs d'un castrum et on peut considérer que l'instauration de vicomtes multiples en Poitou répond moins à une nécessité administrative que militaire et que l'ambitieuse politique comtale qui culmine avec l'appropriation du titre de duc d'Aquitaine vers 965 explique aussi la multiplication des vicomtes en Poitou. La tradition a conservé le souvenir des sept vicomtes de Poitou et a été matérialisée à la fin du XIve siècle par des statues du palais comtal du Poitou ${ }^{66}$.

On sait qu'Alain Barbetorte noua en 942 une étroite alliance avec le duc d'Aquitaine et comte de Poitou, Guillaume Tête d'Etoupe, ce qui lui permit d'obtenir au sud de la Loire trois " pagi ». On peut dès lors concevoir qu'il se soit inspiré de l'organisation mise en place en Poitou. Enfin, il a pu également être influencé par les structures de l'Angleterre anglo-saxonne, qu'il a connues durant son exil Outre-Manche, jusqu'en 936, auprès du roi du Wessex, Athelstan (924-939). L'organisation des royaumes anglo-saxons reposait sur l'existence de circonscriptions, shires équivalents des comtés carolingiens, et dirigés par des earls qui étaient les fondés de pouvoir régionaux des rois; Athestan impulsa un mouvement de renforcement du pouvoir régional entre les mains de quelques grands earls : l'organisation de la puissance publique était donc déconcentrée ou territorialisée ${ }^{67}$.

65. VIADER, Roland, "Conclusions ", dans DeBAX, Hélène (dir.), Vicomtes et vicomtés dans l'Occident médiéval..., op. cit., p. 324.

66. Damon, Géraldine, "Vicomtes et vicomtés dans le Poitou médiéval (IX ${ }^{\mathrm{e}}-\mathrm{XII}{ }^{\mathrm{e}}$ siècle) Genèse, modalités et transformations ", dans DEBAX, Hélène (dir.), Vicomtes et vicomtés dans l'Occident médiéval..., op. cit., p. 224-229.

67. LEBECQ, Stéphane, Histoire des îles britanniques, Paris, PUF, 2007, p. 162-164 : "Au cours du IX ${ }^{\mathrm{e}}$ siècle, une tradition était apparue, celle des 7 rois de l'Angleterre anglosaxonne, qui fut reprise dans la Chronicle du $\mathrm{x}^{\mathrm{e}}$ siècle en y ajoutant un $8^{\mathrm{e}}$ roi, Egbert, roi des West-Saxons (Wessex), avec le qualificatif d'“overlord" (chef suprême) ", ID., ibidem, p. 81-83. 
La question de la postérité des lignages (vicomtaux ou non) qui ont pu être associés à un kemenet, aux $\mathrm{X}^{\mathrm{e}}$ et $\mathrm{XI}^{\mathrm{e}}$ siècles, a été posée dans ce rapide Tour de Bretagne, qui se prolonge par une étude plus approfondie du Kemenet-Ily, au diocèse de Léon.

\section{Du Kemenet-Ily à la vicomté de Léon}

\section{Origines du Kemenet-Ily}

Sa création est postérieure à la Vita de saint-Paul Aurélien (884) qui ne cite que deux pagi, le "pagus Achmensis " et le "pagus Leonensis" (dont la limite commune était la rivière de l'Aber-Wrach, depuis son embouchure jusqu'à sa source à Saint-Thonan, puis le ruisseau de la Pène aboutissant à Beuzit-Conogan, sur l'Elorn), et antérieure à celle de saint Judicaël (datable du $\mathrm{XI}^{\mathrm{e}}$ siècle) qui le mentionne à propos de la paroisse de Tréflez : « in confinum pagi Leonium et Commendationis Illi. " Il a été constitué aux dépens du pagus Leonensis entre ces deux dates et était délimité au nord par la Manche, à l'ouest par la frontière entre les deux pagi primitifs (l'Aber-Wrach et le ruisseau de la Pène) et à l'est par la rivière de la Flèche qui séparait la paroisse de Tréflez de celle de Plounévez-Lochrist. Le territoire du KemenetIly avait pour limite méridionale le cours de l'Elorn, depuis le ruisseau de la Pène, aboutissant à Beuzit-Conogan, et devait donc inclure Landerneau ${ }^{68}$. Il est devenu celui de l'archidiaconé du même nom, tel qu'il ressort du pouillé de l'archevêché de Tours, rédigé vers 1330 pour recenser les églises et prieurés de l'ensemble des diocèses relevant de la province ecclésiastique de Tours : il comprenait seize bénéfices, à l'exclusion de la paroisse de Saint-Houarnon, et du prieuré de Beuzit, qui à cette époque appartenaient à l'archidiaconé d'Ach. À l'inverse, on constate que les paroisses de Landéda et de Lannilis, situées à l'ouest de l'estuaire de l'Aber-Wrach, faisaient partie de l'archidiaconé de Quéménedily. Au sud de l'Elorn, Ploudiry dépendait de l'archidiaconé de Léon ${ }^{69}$.

L'archidiaconé de Quéménedily était donc l'un des trois archidiaconés du diocèse de Léon, dans le Pouillé de la Province ecclésiastique de Tours, et on peut ainsi reconstituer, à partir de la liste des paroisses, la configuration de cette entité géographique, qui a pu varier, car l'église de Saint-Houardon et donc la cité de Landerneau n'en faisaient pas partie vers 1330. Le proto-fief de Kemenet-Ily doit tenir son nom de son premier titulaire, qui pourrait être le vicomte Hily, connu par la charte de l'abbaye de Redon, datable du deuxième tiers du $\mathrm{x}^{\mathrm{e}}$ siècle (voir supra), mais également le vicomte mentionné dans le nécrologe de Landévennec dans lequel est enregistré le décès d'un "vicomte $Y l i$ ", sans date ${ }^{70}$. Ce proto-fief a pu

68. CoufFon, René, « Les "pagi " de la Domnonée au IXe siècle d'après les hagiographes bretons ", Mémoires de la Société d'histoire et d'archéologie de Bretagne, t. XXVI, 1944, p. 3-8.

69. Longnon, Augustin, Pouillé de la province de Tours, op. cit., p. 333-338.

70. Nécrologe de Landévennec, publié par Deuffic, Jean-Luc, Britannia ChristianaBretagne monastique, Landévennec, Fasc.3/1, s.l., 1983, p. 6. 
être constitué après que les Normands aient été vaincus par le duc Alain Barbetorte, vers 936.

\section{Géographie féodale du Kemenet-Ily}

Outre Landerneau, que nous avons considérée comme incluse primitivement dans le Kemenet-Ily, la cité de Lesneven retient l'attention. Géographiquement, elle est située sur une hauteur, au centre du KemenetIly, au carrefour de plusieurs voies, et le préfixe Lez ne peut que conforter l'hypothèse d'un siège seigneurial, fondé par un personnage appelé Neven. Ce nom rappelle celui du comte Even, présenté dans deux notices du cartulaire de Landévennec comme donateur des églises de Lanrivoaré et Lanneufret ${ }^{71}$. Le comte Even est également mentionné dans la vita de saint Goulven, qui relate son combat victorieux contre les Normands ${ }^{72}$, que la tradition place au lieu appelé Runeven, en Plouider, à quelques kilomètres de Lesneven. Cependant, étymologiquement, c'est le nom Neven qui est l'éponyme de Lesneven, personnage qui est évoqué dans une gwerz locale. Faut-il penser qu'Even serait une mauvaise graphie de Neven, anthroponyme moins fréquent? Quoiqu'il en soit, il est difficile de proposer une chronologie pour les événements auxquels est associé le comte Even (ou Neven) : nous pouvons suggérer une hypothèse, en notant que dans la charte de l'abbaye de Saint-Georges de Rennes relatant la donation de l'église de Plougasnou (" in pago Leonensi ") figure parmi les témoins, aux cotés du duc Conan II, "Alanus, filius Ewanni », sans titre, étant précisé que cet acte est daté de $1040^{73}$, ce qui situerait Ewann/Ewen vers l'an mille et en ferait donc un possible successeur du vicomte Ily.

L'histoire de Lesneven est mieux connue à la fin du XII ${ }^{\mathrm{e}}$ siècle, car à cette époque elle constituait une cité seigneuriale des vicomtes de Léon. En effet, la cité de Lesneven apparaît dans les sources écrites dans une charte de 1191 de l'abbaye de Saint-Sulpice-la-Forêt, près de Rennes, relatant la donation par Guiomarch, vicomte de Léon, en faveur du couvent de Locmaria de Quimper d'une maison située à proximité de la chapelle élevée par ses prédécesseurs ${ }^{74}$. Cette chapelle était appelée Notre-Dame de Lesneven, et c'était un prieuré de moniales qui fut rattaché à l'abbaye rennaise, comme Locmaria-Quimper, après 1124. Selon une tradition, ce serait Alain Fergent, duc de Bretagne, qui aurait fondé en 1111 dans le château de Lesneven une

71. Cartulaire de Landévennec, op. cit., p. 568-569.

72. DE LA BORDERIE, Arthur, "Saint Goulven : texte de sa vie latine ancienne, avec notes et commentaires historiques ", Mémoires de la Société d'Emulation des Côtes-du-Nord, t. XXIX, 1891, p. 214-250 (et p. 233-237 et 242-244).

73. Cartulaire de l'abbaye de Saint-Georges de Rennes, publié par DE LA Bigne-VILLENEUVE, Paul, extrait de Bulletin et mémoires de la Société archéologique du Département d'Ille-etVilaine, Rennes, 1876, p. 246-247 : toutefois, ce nom a été ajouté dans la copie du Xve siècle du cartulaire.

74. Dom ANGer, "Cartulaire de l'abbaye de Saint-Sulpice-la-Forêt ", Bulletin et mémoires de la Société archéologique du département d'Ille-et-Vilaine, t. xxxv, 1906, p. 380. 
église Notre-Dame ${ }^{75}$. On peut déduire de cet acte que les vicomtes de Léon étaient possessionnés à Lesneven dès avant 1191 (la chapelle Notre-Dame ayant été fondée par les ancêtres de Guiomarch). Le château, vraisemblablement bâti au XIII ${ }^{\mathrm{e}}$ siècle et encore visible sur le plan cadastral de 1815, paraît avoir succédé à un autre ouvrage dont le parcellaire urbain conserve le tracé et qui se trouvait en haut de la ville actuelle ${ }^{76}$. La cité de Lesneven a conservé sa prééminence en étant le siège de la sénéchaussée de Léon, jusqu'au XVIII ${ }^{\mathrm{e}}$ siècle, où s'exerçait, entre autres, la juridiction des Réguaires de l'évêché de Léon en "Quiminidilly " ${ }^{77}$. La question qui reste posée est celle du lien éventuel entre le vicomte Ily (et éventuellement le comte Even/ Néven) et les grands lignages du Léon.

\section{La question des origines des vicomtes de Léon}

Au cours du XI ${ }^{\mathrm{e}}$ siècle, plusieurs seigneurs peuvent être associés à ce lignage. En premier lieu, un vicomte Guiomarch, dont la mort est relatée dans un extrait du Chronicon Brittanicus, à l'année $1103^{78}$ (" Guichomarius vicecomes Leonensis "), qui eut pour fils Hervé, vicomte, fondateur du prieuré Saint-Martin de Morlaix en faveur de l'abbaye tourangelle de Marmoutiers en 1128. Ce Guiomarch pouvait être le fils ou petit-fils du vicomte Guiomarch, connu vers le milieu du XI ${ }^{\mathrm{e}}$ siècle par des chartes cornouaillaises. Concernant ce vicomte, nous avons développé l'hypothèse selon laquelle il aurait été implanté en Cornouaille, en particulier dans le fief de Kemenet, implantation dont la légende du roi Guinvarch ou Guivarch, collectée à Penhars et à l'île Chevalier, près de Pont L'Abbé, conserverait le souvenir (et que semble corroborer une enquête de 1410-1411 stipulant que « les héritages que le dit vicomte de Rohan - successeur des vicomtes de Léon- tient au Kemenet et en la paroisse de Penharz furent de l'héritage du sire de Pont-L'Abbé ${ }^{79}$ »). Le vicomte Guiomarch pourrait faire partie de la descendance du vicomte Dilès, dont il aurait hérité du titre de vicomte et d'une partie du Kemenet originel, qui aurait été démembré entre ce lignage vicomtal, les comtes de Cornouaille et les seigneurs du Pont ${ }^{80}$. On a constaté enfin que dans l'acte de donation de l'église de Plougasnou en

75. OGÉE, Dictionnaire historique et géographique de Bretagne, nouvelle éd. Revue et augmentée par. MARTEVILLE, André et VARIN, Pierre, Rennes, 1843-1853, t. I, p. 502-503.

76. KERNEVEZ, Patrick, Les fortifications médiévales du Finistère Mottes, enceintes et châteaux, Rennes, Collection Patrimoine Archéologique de Bretagne, 1997, p. 103-105; carte p. 104.

77. BouRdE DE LA RogERIE, Henri, «Liste des juridictions exercées au XVII ${ }^{\mathrm{e}}$ et au XVIII ${ }^{\mathrm{e}}$ siècle dans le ressort du Présidial de Quimper ", Bulletin de la Société archéologique du Finistère, t. XLI, 1914, p. 13.

78. MoRice, Hyacinthe, Mémoires pour servir de preuves à l'histoire ecclésiastique et civile de Bretagne, Paris, 1742-1746, t. I, col. 5.

79. Le Moigne, Gérard, "Le château de Pont L'Abbé », Bulletin de la Société archéologique du Finistère, t. cxxxI, 2002, p. 186-189.

80. Soubigou, Jean-Paul, "Les origines du Kemenet de Cornouaille (IX ${ }^{\mathrm{e}}-\mathrm{XI}^{\mathrm{e}}$ siècles) ", art. cit, p. 113. 
faveur de l'abbaye de Saint-Georges de Rennes ${ }^{81}$, daté de 1040, ne figure pas le nom de Guiomarch, qui vivait vers le milieu du $\mathrm{Xl}^{\mathrm{e}}$ siècle et dont la présence lors de cette donation aurait été logique, s'il avait été vicomte de Léon.

Un autre vicomte aurait porté le titre de vicomte de Léon, Morvan, si l'on se fie à P. Le Baud qui, relatant des luttes entre le duc Conan II et Rivallon de Dol-Combourg, en 1065, cite le nom de Morvan, vicomte de Léon, parmi les alliés du duc ${ }^{82}$. Cet auteur, qui écrivit une Histoire de Bretagne à la fin du $\mathrm{XV}^{\mathrm{e}}$ siècle, rapporte également que, en remerciement, Conan ordonna à Judicael, abbé de Saint-Méen, la restauration de la cité de Brest et d'y édifier une église en l'honneur de la Trinité, de la Vierge et de saint Mathieu. C'est la thèse que les historiens mauristes du XvIII ${ }^{\mathrm{e}}$ siècle ont reprise. Ainsi, H. Morice, dans une note sur la lettre du pape Léon IX adressée en 1049 aux princes de Bretagne, indique que les seigneurs concernés étaient "Alain Canhiart, Morvan, Josselin et Mathias, qui tenaient les comtés de Cornouaille, de Léon, de Porhoët et de Nantes ${ }^{83}$ ". De même, G.-A. Lobineau rapporte que " Ehuarn, Vicomte de Léon, fut père de Guiomarc, qui porta dans le onzième siècle la qualité de vicomte, en même temps que Morvan, que l'on nomme aussi Vicomte de Léon [...] et on peut regarder celui-ci comme la tige des seigneurs du Fou, famille où le nom de Morvan a été très commun ${ }^{84} "$.

Les vicomtes de Léon, à partir de la fin du Xl ${ }^{\mathrm{e}}$ siècle, seraient donc issus Morvan et de Guiomarch, conformément aux affirmations de P. Le Baud et de $\mathrm{H}$. Morice, mais il y aurait eu alliance entre ces deux vicomtes ${ }^{85}$. Les vicomtes du Faou, qui seraient donc issus également de cette alliance, établirent leur pouvoir dans le pagus, d'où ils tirèrent leur nom au $\mathrm{XII}^{\mathrm{e}}$ siècle, en faisant de la cité du Faou le siège de leur fief. Le nomen Ehoarn / Hoarnveus fut réservé aux vicomtes de Léon (en alternance avec Guiomarch), tandis que celui de Morvan se retrouva fréquemment dans le lignage du Faou, de même que Gradlon. Ainsi, dans le dernier tiers du $\mathrm{XI}^{\mathrm{e}}$ siècle, le lignage vicomtal de Morvan aurait imposé son pouvoir sur le Léon à partir de la forteresse de La Roche-Morvan (bâtie sur un éperon rocheux en amont de Landerneau), puis de Lesneven, au détriment des primates leonenses (l'aristocratie du Léon). À l'origine, il devait exercer la charge de vicomte auprès du comte Budic et de ses successeurs, et devait être primitivement possessionné dans le "pagus ", entre l'Aulne et les Monts d'Arrée ${ }^{86}$. Cette thèse de l'origine cornouaillaise du lignage vicomtal, au pouvoir en Léon à partir du

81. Cartulaire de l'abbaye de Saint-Georges de Rennes, op. cit., p. 246-247.

82. LE BAUD, Pierre, Histoire de Bretagne avec les Chroniques des Maisons de Laval et de Vitré, Paris, éd. P. d'Hozier, 1638, p. 156-157.

83. Morice, Hyacinthe, TAIILLANDIER, Charles, Histoire ecclésiastique et civile de Bretagne, Paris, 1750-1756, t. I, p. 983.

84. LoBINEAU, Guy-Alexis, Histoire de Bretagne, Paris, 1707, livre III, p. 106.

85. Voir Annexe 1.

86. SouBigou, Jean-Paul, "Le vicomte Morvan, entre Léon et Cornouaille (XI ${ }^{\mathrm{e}}$-XII ${ }^{\mathrm{e}}$ siècles) ", Bulletin de la Société archéologique du Finistère, t. cxxxvII, 2008-2009, p. 255-271. 
$\mathrm{XI}^{\mathrm{e}}$ siècle, a été proposée ou évoquée par quelques auteurs ${ }^{87}$. Les vestiges du château de La Roche-Morvan (commune de La Roche-Maurice) ont fait l'objet d'une campagne de fouilles archéologiques, entre 2002 et 2007, qui a révélé cinq phases d'occupation entre le $\mathrm{xl}^{\mathrm{e}}$ et le $\mathrm{XV}^{\mathrm{e}}$ siècle ${ }^{88}$. Il a vraisemblablement été édifié par le vicomte Morvan vers le milieu du XI ${ }^{\mathrm{e}}$ siècle et a appartenu aux vicomtes, puis aux seigneurs de Léon, jusqu'au XIV ${ }^{\mathrm{e}}$ siècle : n'est-il pas le symbole de la continuité d'un même lignage vicomtal?

\section{Nouvelles perspectives}

Si aucun de ces deux lignages vicomtaux ne peut être présenté comme descendant du vicomte Ily, il convient de rechercher dans l'aristocratie léonarde si des familles nobles peuvent être rattachées au premier titulaire du Kemenet-Ily.

\section{De Carman, ancien lignage du Léon}

Parmi les grandes maisons du Léon, c'est la seigneurie de Carman qui était la mieux implantée dans le Kemenet-lly : à la fin de l'Ancien-Régime elle était le deuxième " ménéant " de la sénéchaussée de Lesneven, après la principauté de Léon. La juridiction exercée à Kernilis, puis à Plouguerneau et Lannilis, avait dans son ressort, outre les trois paroisses précitées, celles de Kerlouan, Guissény, Landéda, Plouvien, Plouider, Plounéour et LocBrévalaire ${ }^{89}$. Cette juridiction recouvrait donc un territoire correspondant au nord de l'archidiaconé de Kemenet-lly. Le nom de ces seigneurs, considérés comme l'une des quatre plus grandes familles de l'évêché de Léon ${ }^{90}$, vient du château, aujourd'hui disparu, qu'ils édifièrent sur la paroisse de Kernilis, au lieudit Kermaguam ("Kervaon " en breton), qui a évolué en Kermavan et Carman, en français. D'après une légende, un souterrain aurait relié ce château à celui de Lesneven, distant d'une dizaine de kilomètres.

87. Everard, Judith, Britanny and the Angevins : Province and Empire : 1158-1203, Cambridge, 2000, p. 18 et 19 : cette historienne établit des liens de vassalité des vicomtes de Léon envers les comtes de Cornouaille; MoRvan, Frédéric : "Le Léon féodal au XIII ${ }^{\mathrm{e}}$ siècle ", Mémoires de la Société d'histoire et d'archéologie de Bretagne, Actes du Congrès de Brest, t. Xc, 2012, p. 39 : c'est l'une des hypothèses évoquées par l'auteur quant à l'origine de ce lignage vicomtal.

88. "Le château de La Roche-Maurice " : compte-rendu de l'excursion du 22 mai 2012, sous la direction de Kerherve, Jean, et Kernevez, Patrick, Bulletin de la société archéologique du Finistère, t. cXL, 2012 : p. 15 à 17.

89. BouRdE DE LA ROGERIE, Henri, "Liste des juridictions exercées aux XVII ${ }^{\mathrm{e}}$ et XVIII ${ }^{\mathrm{e}}$ siècles dans le ressort du Présidial de Quimper ", Bulletin de la Société archéologique du Finistère, t. XLI, 1914, p. 16.

90. Un dicton le rappelle : "Antiquité de Penhoët, vaillance du Chastel, richesse de Kermavan et chevalerie de Kergournadec'h ", cité par FlaGELLE, Émile, " Notes archéologiques sur le département du Finistère ", Bulletin de la Société académique de Brest, t. IV, 1876-1877, p. 78. 
Au XIII ${ }^{\mathrm{e}}$ siècle, les seigneurs de Kermavan annexèrent la seigneurie de Seizploué, située à Plounévez-Lochrist, et on trouve dans l'église priorale de Lochrist une pierre tombale portant l'épitaphe d'Alain de Kermavan, mort en $1263^{91}$. C'est sans doute son fils, Alain de Kermavan qui est mentionné dans le Livre des Ost, en 1294, juste après Hervé de Léon. Ce document permet de connaître les plus grandes familles de la noblesse à la fin du XIII ${ }^{\mathrm{e}}$ siècle, et pour le Léon il n'en recense que six : outre les deux précitées, on relève le vicomte du Faou, pour sa terre de Léon, Alain Nuz (Kergoudanec'h), Bertrand de Kernau (Cornouaille?) et Hervé du Chastel ${ }^{92}$.

Le fief possédé par le vicomte du Faou pourrait être le manoir du VieuxChastel, situé sur la paroisse de Taulé, et qui appartenait au Xve siècle à une branche de la famille du Faou ${ }^{93}$ (l'implantation des vicomtes du Faou en Léon pourrait résulter d'un partage entre les deux lignages vicomtaux issus de Ehuarn : voir supra). Le siège du fief d'Alain Nuz devait être le château de Tournus à Plounévez-Lochrist, en l'archidiaconé de Léon; quant au lignage de Kernau-Cornouaille, le Livre des Ost indique "pour son fief de Kerguerne ", qui pourrait être identifié, comme étant la seigneurie de Kervern en Guimiliau ${ }^{94}$, ou de Kerguern en Guipavas; enfin, le château de Trémazan en Landunvez était le centre des fiefs des Du Chastel ${ }^{95}$. On voit donc que le lignage de Kermavan était le plus puissant en Kemenet-Ily et qu'il pourrait remonter aux premiers temps de ce fief. La localisation du château à Kervaon en Kernilis, au fond de l'estuaire de l'Aber-Wrach et non loin de l'antique voie reliant Lesneven à Plouguerneau, pourrait indiquer que le lignage s'est excentré.

\section{Vicomtes en Kemenet-Ily}

En Kemenet-Ily, deux maisons nobles ont porté le titre de vicomte jusqu'à la fin du Moyen Âge. Celle de Coat-Menech se disait "vicomte de Plouider " jusqu'au XVIII ${ }^{\mathrm{e}}$ siècle et le ressort de cette seigneurie s'exerçait à Guissény, Plouguerneau Kernilis, Kerlouan et Plouider ${ }^{96}$. C'était sur cette dernière paroisse limitrophe, au nord-est, de Lesneven, que se trouvait le siège de son fief, près de Coat-Menac'h-Bihan, où une parcelle est appelée " Goarem-ar-Hastel " (garenne du château). Ce lignage est attesté depuis

91. Simon, Marc, «En Plounévez-Lochrist, un vénérable sanctuaire », Pax, Landévennec, $\mathrm{n}^{\circ}$ 95, juillet 1973, p. 87 .

92. Morvan, Frédéric "Le "Livre des Ostz" (1294). Un éclairage sur les rapports du duc avec la noblesse bretonne à la fin du XIII ${ }^{\mathrm{e}}$ siècle, dans KERHERVÉ, Jean (dir.), Noblesse de Bretagne du Moyen Âge à nos jours, (Actes de la journée d'études à Guingamp, le 29 novembre 1997), Rennes, PUR, 1999, p. 85.

93. LE Guennec, Louis, "Le chemin du Tro-Breiz entre Saint-Pol-de-Léon et Tréguier ", Bulletin de la Société archéologique du Finistère, t. XxxIII, 1906, p. 259.

94. YeURC'H Bertrand, message du 22 mars 2012, dont je le remercie sincèrement.

95. Morvan, Frédéric, Le « Livre des Ostz »..., op. cit., p. 85-86.

96. BOURDE DE LA ROGERIE, Henri, « La liste des juridictions exercées aux XVII ${ }^{\mathrm{e}}$ et XVIII ${ }^{\mathrm{e}}$ siècles dans le ressort du Présidial de Quimper ", art. cit, p. 22. 
1373 au moins ${ }^{97}$. En outre, elle avait la charge honorifique d'être l'une des quatre nobles du Léon à porter la chaise de l'évêque lors de son intronisation en la cathédrale de Saint-Pol-de-Léon, avec les seigneurs de Kermavan, de Coetivy et de Kervern ${ }^{98}$.

L'autre famille avait le centre de son fief sur la paroisse de Plouguerneau. Mais, il convient de signaler tout d'abord les toponymes Treffily, à Lannilis, et Kerily, à Plouguerneau. Le premier est connu par la famille de Treffily, qui remonterait à 1248 et possédait des biens à Lannilis en 1407. Le second est un lieudit de la paroisse de Plouguerneau, qui a donné son nom à une famille noble mentionnée en $1447^{99}$. Le premier de ces toponymes désignant à partir du XI ${ }^{\mathrm{e}}$ siècle un quartier de paroisse et le second un hameau ${ }^{100}$, ils peuvent avoir un lien avec le vicomte Ily, qui vivait vers 950. Ainsi, dans ces deux paroisses de l'archidiaconé de Kemenet-Ily, des terres nobles pourraient avoir porté le nom d'un personnage appelé Ily.

Or, Kerily est tout proche du manoir de Coat-Quénan, dont les seigneurs portaient le titre de vicomte au Xve siècle, selon plusieurs sources ${ }^{101}$. Le manoir de Coat Quénén, qui fut vraisemblablement précédé d'une motte féodale, existe encore : la présence de douves et le toponyme "Porz-al-Lez " (la cour de la résidence seigneuriale) indiquent l'importance et l'ancienneté du site; en outre, les possesseurs de cette vicomté auraient détenu le droit de haute justice et se prévalaient d'être seigneurs fondateurs de l'église paroissiale de Plouguerneau ${ }^{102}$. Le manoir est distant de deux kilomètres environ de l'antique Pont-Crach, qui permettait le passage de l'Aber-Wrach vers Lannilis, d'une part, et de trois kilomères environ du site de Carman, où s'élevait la forteresse des seigneurs de Carman, d'autre part. Vers 14001426, la vicomté de Coat-Quénan, dont la juridiction s'étendait sur plusieurs paroisses (Plouguerneau, Landéda, Kernilis et Plounéour-Trez), était entre les mains de Blanche de Cornouaille, qui dans un aveu de 1426 stipulait posséder cinq autres manoirs, dont celui de Grouannec ${ }^{103}$. Le titre de vicomte a été porté par la famille de Parcevaux, puis par celle de Carné, qui héritèrent du fief de Coat-Quénan aux XVI ${ }^{\mathrm{e}}$ et XVII ${ }^{\mathrm{e}}$ siècle $^{104}$. Ainsi, cet ancien lignage du Léon, portant le titre de vicomte, pourrait apparaître comme descendant du vicomte Ily, étant implanté anciennement dans le Kemenet Ily, comme en témoigne l'existence d'un site castral à Coat-Quénan. Une origine commune

97. KeRnEVEZ, Patrick, Les fortifications du Moyen Âge dans le Finistère, op. cit., p. 149.

98. YEURC'H, Bertrand, message du 25 mars 2012, dont je le remercie sincèrement.

99. TORCHET, Hervé, Réformations des fouages de 1426 : Diocèse ou évêché de Léon. Notices biographiques et généalogiques, introduction d'A. DESHAYES, notices biographiques et généalogiques par H. TORCHET, illustrations héraldiques par A. CHAUVEL, Paris, Éditions La Pérenne, 2010, p. 275 et 213.

100. TAnguY, Bernard, Dictionnaire des paroisses..., op. cit., p. $26-29$.

101. Torchet, Hervé, Réformations des fouages de $1426 \ldots$. op. cit., p. 17.

102. KernEvez, Patrick, Les fortifications du Moyen Âge dans le Finistère, op. cit, p. 147.

103. Perennes, Henri, Plouguerneau (Une paroisse entre Manche et Océan), LangonnetQuimper, Imprimerie de l'orphelinat Saint-Michel, 1941, p. 21.

104. De KeRLIVER, René, Répertoire général de bibliographie bretonne, Rennes, 1898, t.10, p. 24 . 
avec le puissant lignage de Carman pourrait être suggérée, en raison de la proximité des deux fiefs.

\section{Tentative de chronologie}

- le vicomte Ily aurait été nommé à la tête du Kemenet de Léon avant 952, date du décès d'Alain Barbetorte;

- le " comte Even/Néven" a pu être son successeur : sa victoire contre les Normands est relatée brièvement par Flodoard, auteur de la Chronique des Francs (919-966) ${ }^{105}$;

- on peut signaler "Alain, fils d'Ewan ", en 1040, dans la charte de donation de l'église de Plougasnou en faveur de l'abbaye de Saint-Georges de Rennes;

- après la victoire d'Alain Canhiart, comte de Cornouaille, au milieu du $\mathrm{XI}^{\mathrm{e}}$ siècle, sur les Léonais à propos des limites territoriales ("limes "), le vicomte Morvan, témoin de la charte donnée par le comte à cette occasion en faveur de l'évêché de Cornouaille ${ }^{106}$, a pu investir une partie du Léon et édifier au bord de l'Elorn la forteresse qui prit le nom de La Roche-Morvan, près de Landerneau;

- vers la fin du deuxième tiers du XI ${ }^{\mathrm{e}}$ siècle, il a pu avoir la mainmise sur le Kemenet-lly (Lesneven) et l'ouest du Léon, jusqu'à Brest, qui fut fortifiée à la demande du duc Conan II, en contrepartie du soutien qu'il lui apporta vers 1065 ;

- Ehuarn, fils du vicomte Morvan, puis Guiomarch, vicomte de Léon, imposèrent le pouvoir de leur lignage sur l'ensemble du Léon, jusqu'à Morlaix, et ce dernier fut tué par ruse en 1103, ce qui tendrait à montrer qu'il se heurtait encore à l'hostilité d'une partie de l'aristocratie léonarde.

- nous avons envisagé que la famille de Kermavan serait un rameau issu du premier détenteur du pouvoir en Kemenet-Ily, mais le titre de vicomte ne lui est pas resté. Il pourrait avoir été détenu par la famille de Coat-Quénan, implantée à Plouguerneau depuis le XIV ${ }^{\mathrm{e}}$ siècle au moins. Ainsi, de même qu'en Poudouvre, où les seigneurs de Dinan furent la puissance dominante, mais sans le titre vicomtal, détenu par les vicomtes de Poudouvre, puis à la fin du XIII ${ }^{\mathrm{e}}$ siècle par la famille de la Bellière, le lignage de Kermavan était fortement implanté en Kemenet Ily, mais le titre vicomtal serait revenu aux seigneurs de Coat Quénan, qui le conservèrent jusqu'au Xve siècle au moins.

L'implantation du lignage vicomtal de Léon dans l'évêché dont il a repris le nom peut être comparée à celle de la branche cadette des comtes de Rennes, représentée par Eudon, frère du duc Alain III, qui imposa son pouvoir dans les diocèses de Tréguier (au détriment des vicomtes de

105. LA BorDERIE, Arthur de, Histoire de Bretagne, t. II, Rennes/Paris, 1898-1899, p. 390-391. 106. Morice, Hyacinthe, Mémoires..., op. cit., t. I, col. 377. 
Pommerit) et de Saint-Brieuc, et qui fortifia les cités de Guingamp et de Lamballe, à partir des années $1040^{107}$. Vers la même époque en effet, le lignage vicomtal de Léon, qui serait issu des vicomtes de Cornouaille, aurait supplanté un lignage installé dans le Kemenet-lly, fief vraisemblablement confié au vicomte lly (cité dans une charte du deuxième tiers du $\mathrm{x}^{\mathrm{e}}$ siècle), et il aurait fortifié Les-Neven. En Poudouvre, vicomté dans laquelle la branche aînée continua de porter le titre de vicomte jusqu'à la fin du XII ${ }^{\mathrm{e}}$ siècle, le titre échut ensuite aux vicomtes de La Bellière au XIII ${ }^{\mathrm{e}}$ siècle, alors que les branches cadettes, devenues de puissantes seigneuries, ne portaient plus ce titre. Les premiers vicomtes de Porhoët, au XI ${ }^{\mathrm{e}}$ siècle, pourraient avoir succédé au premier titulaire du Kemenet de Porhoët-en-Vannes, mais le titre de vicomte échut à la branche cadette de Rohan, après 1120. La maison des comtes de Cornouaille, quant à elle, serait issue d'une alliance entre le lignage du vicomte Dilès, possessionné en Kemenet, et celui du comte Budic Castellin, implanté dans la vallée de l'Aulne, et nous avons proposé que le vicomte Guiomarch vivant vers le milieu du $\mathrm{XI}^{\mathrm{e}}$ siècle serait un descendant (issu d'une branche cadette?) du lignage vicomtal de Dilès. Enfin, les seigneurs de Kemenet-Héboé seraient, soit les descendants d'un lignage comtal, proche de Juhel Bérenger, soit issus des vicomtes de Vannes.

La création des Kemenet a constitué, dans la deuxième moitié du $\mathrm{X}^{\mathrm{e}}$ siècle, une transition entre les invasions normandes et l'émergence du système féodal en Bretagne. La fondation de ces proto-fiefs pourrait avoir été engagée par Alain Barbetorte, qui mena une politique extérieure active puisqu'il fit alliance avec le comte d'Anjou, duc d'Aquitaine, et qu'il épousa la sœur du comte de Blois ${ }^{108}$. On peut penser qu'il eut de même l'ambition d'engager une politique volontariste à l'intérieur de la principauté bretonne et qu'il mit en œuvre une nouvelle organisation à la fois institutionnelle et militaire, en attribuant des territoires à des hommes de confiance. Cette politique fut vraisemblablement menée en accord avec le comte de Rennes Juhel Bérenger, avec lequel il fit alliance pour gouverner la principauté bretonne (en 942, Alain prête hommage en même temps que Juhel Bérenger au duc de Normandie, à Rouen ${ }^{109}$ ), et l'influence de Juhel Bérenger pourrait être décelée dans le Kemenet trègorois et dans le Kemenet-Héboé. On a constaté enfin une coïncidence entre les Kemenet et le titre de vicomte, qui traduirait une territorialisation de cette fonction, ce qui va à l'encontre de l'institution carolingienne originelle, reprise dans l'organisation féodale, qui voulait que le vicomte soit l'adjoint du comte, mais sans posséder d'assise territoriale : cette déconcentration du pouvoir comtal se retrouve dans le comté de Poitou, dès la première moitié du $\mathrm{X}^{\mathrm{e}}$ siècle. L'institution spécifique des Kemenet en Bretagne, vers le milieu du $\mathrm{X}^{\mathrm{e}}$ siècle, fut ainsi une nécessité,

107. Аміот, Christophe, "Lignages et châteaux des baillies de Penthièvre et Tréguier du $\mathrm{XI}^{\mathrm{e}}$ au milieu du Xv ${ }^{\mathrm{e}}$ siècle ", Bulletin de la Société d'émulation des Côtes-d'Armor, (2009), t. CXXXVIII, Année 2010, p. 3 : “Il s'agissait de réorganiser des territoires littoraux d'une zone institutionnellement perturbée. "

108. Chedeville, André, et Tonnerre, Noël-Yves, La Bretagne féodale ..., op. cit., p. 27-30.

109. Les annales de Flodoard, Paris, éd. LAUER, Philippe, 1906, p. 84. 
en raison de la désorganisation causée par les invasions scandinaves, et elle ne put que favoriser la politique extérieure du premier duc, qui eut l'ambition de jouer un rôle influent dans la Francia Occidentalis ${ }^{110}$.

À partir de la fin du x $x^{\mathrm{e}}$ siècle, c'est la maison comtale de Rennes qui détint le pouvoir ducal, avec Conan, fils de Juhel Bérenger, et qui le conserva jusqu'à l'accession du comte de Cornouaille, Hoël, en 1066, puis de son fils Alain Fergent, en 1084. Parallèlement, on a constaté l'expansion territoriale de la famille vicomtale issue de Morvan, au cours du troisième tiers du XI ${ }^{\mathrm{e}}$ siècle, qui installa son pouvoir dans le Léon. La participation d'Hervé, fils du vicomte Guiomarch, à la première Croisade, qui débuta en $1096^{111}$, montre que le lignage vicomtal de Léon, à la fin du XI ${ }^{\mathrm{e}}$ siècle, faisait partie des plus grandes familles de l'aristocratie bretonne, aux côtés du duc Alain Fergent, qui était descendant d'Alain Barbetorte. Un siècle et demi après la mort du premier duc, le duché de Bretagne comptait neuf diocèses, dont un archevêché, à Dol-de-Bretagne, et ses limites orientales et méridionales étaient fixées (de Fougères à Clisson) jusqu'à la fin de l'Ancien-Régime.

110. TONNERRE, Noël-Yves, op. cit., p. 289.

111. De LA BORDERIE, Arthur, Histoire de la Bretagne, op. cit., t. III, p. 82 : d'après BAUDRI DE BOURGUEIL, archevêque de Dol-de-Bretagne au XII e siècle : "Hervé, le fils Guiomarch qui lors était vicomte de Léon. " 


\section{Annexe 1 - Essai de reconstitution de la généalogie des vicomtes du Faou et de Léon (XI ${ }^{e}$-XII ${ }^{e}$ siècles)}

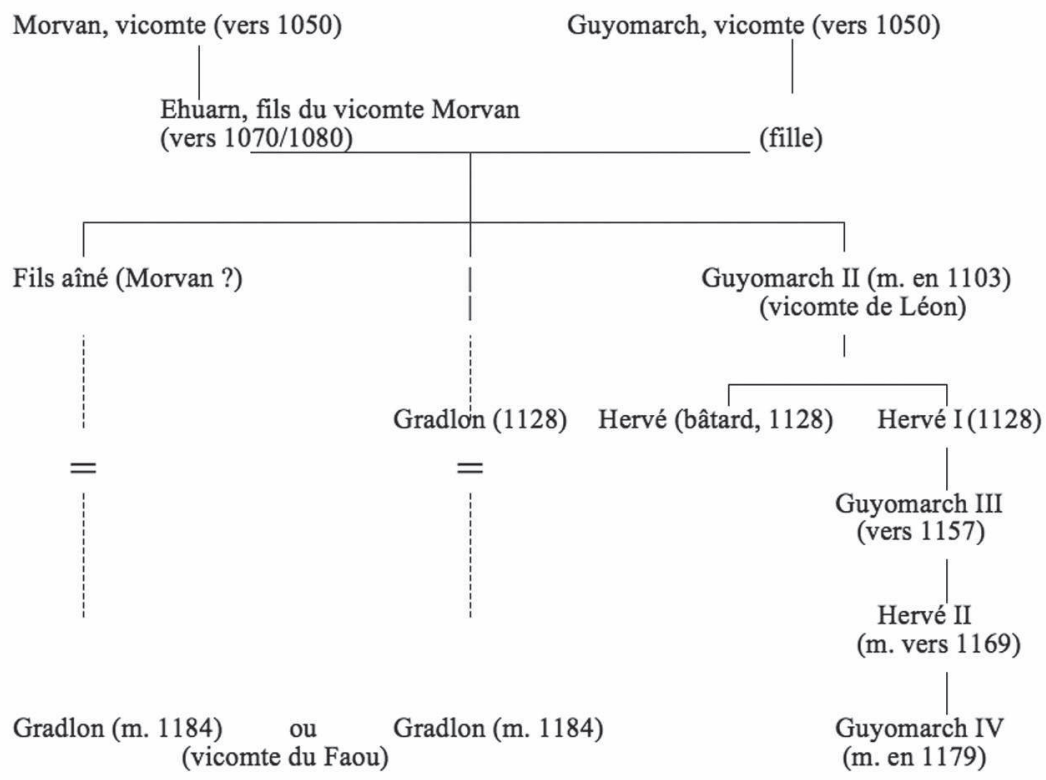

\section{Annexe 2 - L'ancien évéché de Léon : division en trois archidiaconés}

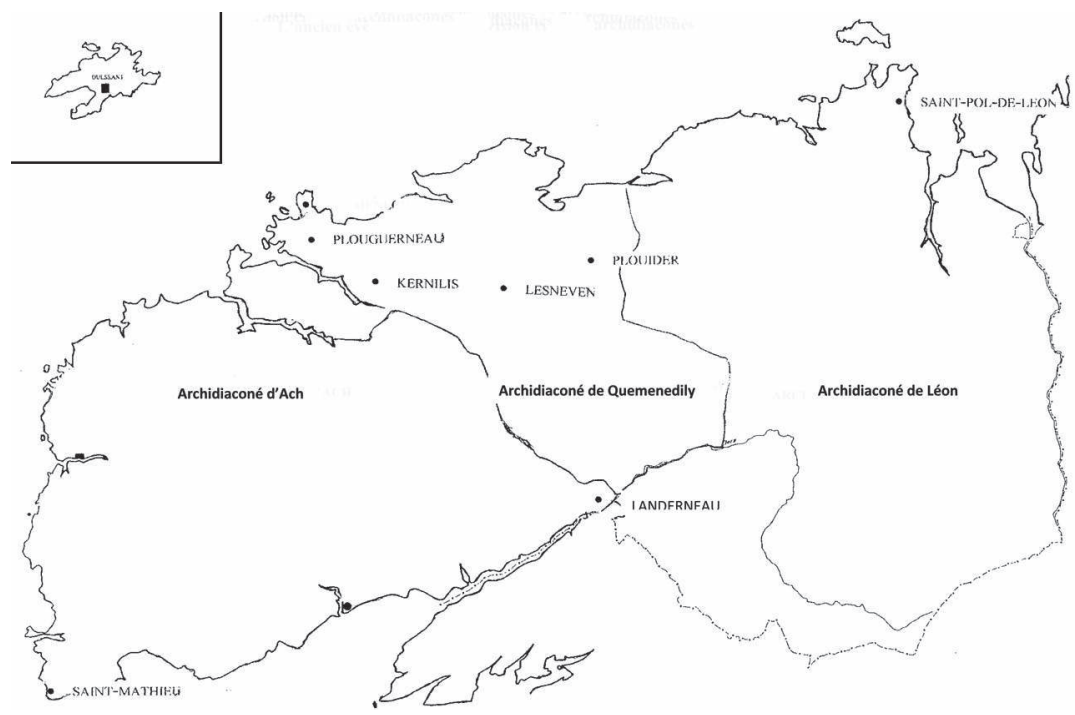




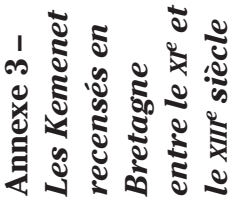

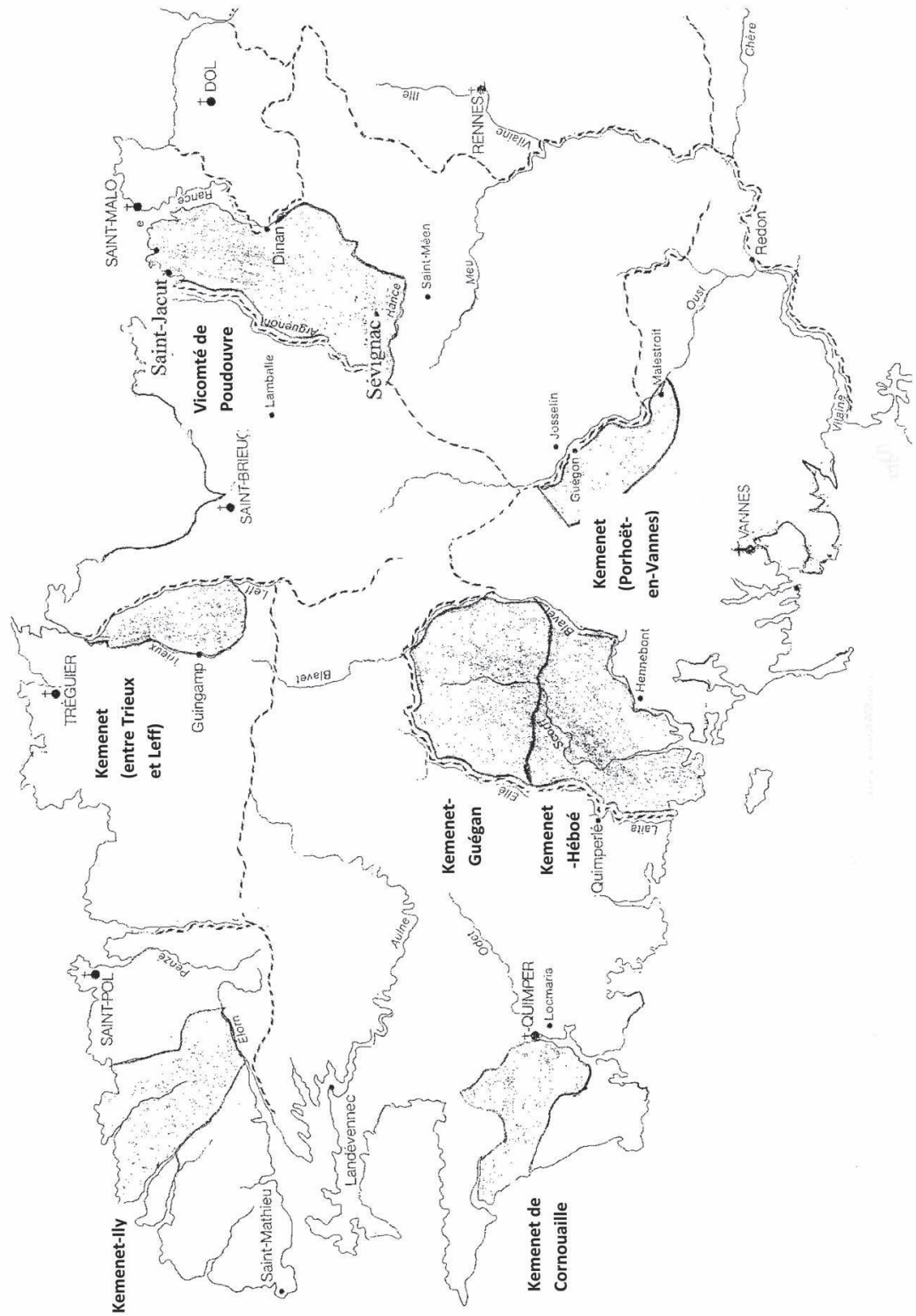




\section{RÉSUMÉ}

L'histoire du Léon s'inscrit, de la fin des invasions normandes, vers 936, jusqu'à la première Croisade, en 1096, dans celle du duché de Bretagne. Le fief de Kemenet-Ily, en Léon, fut l'un des sept Kemenet (en incluant la vicomté de Poudouvre) recensés en Bretagne. Ce mot, désignant en langue bretonne un fief donné à un baron, était déjà cité dans les actes dès la première moitié du XI ${ }^{\mathrm{e}}$ siècle. On peut penser qu'ils furent créés par le duc Alain Barbetorte, vers le milieu du $\mathrm{x}^{\mathrm{e}}$ siècle, afin de restaurer la principauté bretonne, et que certains eurent à leur tête des vicomtes, comme le Kemenet de Cornouaille et le Kemenet-Ily. L'étude cherche à identifier leurs descendants dans des lignages qui portaient encore le titre, devenu honorifique, de vicomte, jusqu'au $\mathrm{Xv}^{\mathrm{e}}$ siècle. Puis, des lignages plus puissants s'imposèrent au $\mathrm{XI}^{\mathrm{e}}$ siècle. Ainsi, la maison vicomtale de Léon, dont les origines seraient cornouaillaises, supplanta l'aristocratie léonarde (" primates leonenses "). Ils édifièrent la forteresse de la Roche-Morvan et renforcèrent les cités de Lesneven, en Kemenet-Ily, puis de Brest et de Morlaix, cette dernière devenant le centre de leur pouvoir jusqu'à la fin du XII ${ }^{\mathrm{e}}$ siècle.

\section{ABSTRACT}

The history of Léon was linked to that of the duchy of Brittany, from the end of Norman invasions (in about 936) up to the first crusade in 1096. The lordship of Kemenet-Ily, in Léon, was one of the seven known Kemenet (including the viscountcy of Poudouvre) in Brittany. A Kemenet was a territory given to a baron, and the word was already employed in deeds in the first half of the eleventh century. They were probably created by Duke Alain Barbetorte in the middle of the tenth century to strengthen the principality of Brittany. Some of their lords were viscounts, as was the case for the Kemenet of Cornouaille and Kement-Ily. This study tries to identify their descendants in lines that bore the title of viscount up to the fifteenth century. Then, more powerful lineages took control during the eleventh century. Thus, the house of the viscounts of Léon, which probably came from Cornouaille, replaced the local aristocracy (the "primates leonenses"). They built the castle of La Roche Morvan and reinforced the city of Lesneven, in Kemenet-Ily, and later those of Brest and Morlaix, the latter town becoming the centre of their power up to the end of twelfth century. 